\title{
Monte Carlo Simulation of Long Chain Polymer Melts: Crossover from Rouse to Reptation Dynamics
}

\author{
T. Kreer ${ }^{1 *}$, J. Baschnagel ${ }^{2}$, M. Müller ${ }^{1}$, K. Binder ${ }^{1}$ \\ ${ }^{1}$ Institut für Physik, Johannes-Gutenberg Universität, D-55099 Mainz, Germany \\ ${ }^{2}$ Institut Charles Sadron, 6 rue Boussingault, F-67083, Strasbourg Cedex, France
}

\begin{abstract}
We present data of Monte Carlo simulations for monodisperse linear polymer chains in dense melts with degrees of polymerization between $N=16$ and $N=512$. The aim of this study is to investigate the crossover from Rouselike dynamics for short chains to reptation-like dynamics for long chains. To address this problem we calculate a variety of different quantities: standard mean-square displacements of inner monomers and of the chain's center of mass, the recently proposed cubic invariant [U. Ebert et al., Phys. Rev. Lett. 78, 1592 (1997).], persistence of bond-vector orientation with time, and the auto-correlation functions of the bond vector, the end-to-end vector and the Rouse modes. This analysis reveals that the crossover from non- to entangled dynamics is very protracted. Only the largest chain length $N=512$, which is about 13 times larger than the entanglement length, shows evidence for reptation.
\end{abstract}

PACS: 61.20.Ja,64.70.Pf,61.25.Hq,83.10.Nn

submitted to Macromolecules on August 24, 2000

Typeset using REVTEX

*To whom correspondence should be addressed. Email: tkreer@plato.physik.uni-mainz.de 


\section{INTRODUCTION}

A detailed description of the slow dynamics of entangled polymer chains would be of great interest for better understanding the physical properties of dense polymer melts or solutions. Several attempts to solve this problem have been made. In a seminal paper de Gennes introduced the concept of reptation [1]. The fundamental idea is that the motion of a polymer is not spatially isotropic, but has to occur along the contour of a "tube" which is formed by the surrounding chains. These surrounding chains are treated as a fixed network of impenetrable obstacles. The simultaneous motion of the polymers and possible correlations resulting from that are disregarded. The elaboration of this idea has led to a rich scenario of testable predictions [2 4. Critical analysis of these predictions by experiments [5 9] and computer simulations [10 12] suggest that reptation is an important relaxation mechanism. However, it cannot provide a quantitative explanation of all dynamic features observed. These deviations could result from additional relaxation processes which blur the clear signature of reptation for typical experimental chain lengths $N$ and become negligible only in the asymptotic limit of extremely large $N$ (see, however, [13] for a different asymptotic behavior).

These additional relaxation mechanisms could involve fluctuations of the tube's contour [14 [16], reorganization of the tube [17] by release and creation of topological constraints [5. 14.18], elastic distortions of the entanglement network [19] or long-lived density fluctuations [20]. All these extensions assume the existence of a tube. None of them explains its microscopic origin. An attempt to develop a microscopic theory was made by the development of a mode-coupling theory for polymers [21 26]. This approach leads to similar predictions as reptation theory without invoking a tube hypothesis.

However, some doubt was cast on this concept by simulations of a model by Shaffer 27 which respects excluded volume interactions between the monomers of the chains, but allows Monte Carlo moves that lead to intersections between bonds connecting the monomers. For long chains in this model a simple Rouse-like behavior is found. Only when the conditions that bonds must not intersect is used as an additional constraint in the dynamics, a reptation-like behavior results from the simulation. These results, where static correlations are precisely identical for both models, but one dynamic version exhibits Rouse behavior and the other reptation-like behavior, seem to imply that the topological constraint of the noncrossability of chains is not an automatic consequence of the excluded volume interaction. In this context, we note that many other attempts have been made to account for the slow dynamics of entangled polymer chains by concepts differing from reptation, e.g., collective motion of many entangled chains [28], but we are not attempting to critically evaluate all these theories here.

In view of these diverse attempts to improve on reptation theory, it is also beneficial to consider a model which exhibits pure reptation behavior in the asymptotic limit of large chains and long times, and for which finite- $N$ and finite-time corrections can be calculated. Evans and Edwards proposed such a model in which a single chain moves through a reg-

ular array of impenetrable obstacles [29]. The obstacles do not influence the equilibrium configuration of the chain, but only the accessible relaxation moves of its monomers. This model has recently been reanalyzed analytically [30,31]. Detailed comparisons with computer simulations [30,32,33] reveal an extremely slow crossover to the asymptotic power laws 
characteristic of reptation. Even in the ideal situation, where the tube diameter is equal to the bond length, the power laws only become clearly pronounced for $N>100$. If the lattice of obstacles is diluted, the tube diameter increases and the crossover to reptation is shifted to larger chain lengths 33 .

The latter study resembles more closely the situation encountered in computer simulations of melts where the tube diameter is in general larger than the bond length. Taking into account that simulations typically work with $N<1000$, the analysis of the EvansEdwards model suggests that the results of polymer melt simulations are characteristic of the crossover from non-entangled, Rouse-like dynamics for short chains to (slightly) entangled, reptation-like dynamics for long chains. With the present study we want to further investigate this crossover by a Monte Carlo simulation of the bond-fluctuation model. Our paper is organized as follows: Section II introduces the model and describes some of its static properties. Section III represents the main part of the paper. It deals with dynamics of the model. Here, we compare various mean-square displacements and orientational correlation functions, extract the chain length dependence of relaxation times and of the diffusion coefficient, and estimate the parameters of reptation theory, such as the tube diameter or the entanglement length. The final section [V] summarizes our conclusions.

\section{MODEL AND STATIC PROPERTIES}

The aim of the present work is to extend and to complement previous studies of the crossover from Rouse to reptation dynamics using the bond-fluctuation model [34 36]. The bond-fluctuation model is a coarse-grained lattice model [12,37,38, in which a polymer is represented by a chain consisting of $N$ monomers. Each monomer occupies a unit cell of a simple cubic lattice (unit cell $=8$ lattice sites). This increased monomer size allows the bond vectors $\boldsymbol{l}$ to vary in both length and direction to a much larger extent than in simpler lattice models where a monomer is associated with a single site 37. In total, there are 108 bond vectors [39] giving rise to 87 bond angles and 5 different bond lengths $(2, \sqrt{5}, \sqrt{6}, 3, \sqrt{10})$. The average bond length and bond angle depend on the volume fraction, $\phi=8 N P / L^{3}$, of occupied lattice sites $(P=$ total number of polymers in the simulation box, $L=$ linear dimension of the box). Choosing $\phi=0.5$ the model realizes typical properties of dense melts [34]. For this density the mean bond length and mean cosine of the bond angle are given by $l=\left\langle\boldsymbol{l}^{2}\right\rangle^{1 / 2}=2.64$ and $\alpha=\langle\cos \theta\rangle=-0.1055$, respectively 40.

To analyze the change in dynamic behavior with increasing $N$ simulations were performed for different chain lengths ranging from $N=16$ to $N=512$ (see Table 凹). This extends previous studies [34] by a factor of 2 only, but it amounts to an increase in relaxation time by almost an order of magnitude (cf. Table 【). Furthermore, the system size could also be chosen much larger. For most chain lengths the linear dimension of the simulation box is more than a factor of 2 greater than the average end-to-end distance $R_{\mathrm{e}}$, i.e., $L>2 R_{\mathrm{e}}$ (with the exception of $N=512$ where $L=128 \simeq 1.75 R_{\mathrm{e}}$ only; see Table (I). Since finite-size effects may be expected if $L \leq 2 R_{\mathrm{e}}$, Fig. 目 compares the mean-square end-to-end distance $R_{\mathrm{e}}^{2}$ and

mean-square radius of gyration $R_{\mathrm{g}}^{2}$ of the present study with the values of earlier works [34,35]. Two conclusions can be drawn from this figure. First, finite-size effects did not affect previous (static) results. Within the statistical uncertainties the old data for $N=200$ $\left(R_{\mathrm{e}} \simeq 45, L=40\right.$ [34]) agree with the present analysis. Second, the bond-fluctuation model 
approaches the asymptotic ideal chain limit, i.e., $R_{\mathrm{e}}^{2}=6 R_{\mathrm{g}}^{2}=C_{\infty} l^{2}(N-1)$, if $N>100$. Here, $C_{\infty}\left(=\lim _{N \rightarrow \infty} R_{\mathrm{e}}^{2} /\left[(N-1) l^{2}\right]\right)$ is the characteristic ratio which measures the swelling of a chain due to its intrinsic stiffness compared to a pure random walk. Using $C_{\infty} \simeq 1.56$ (see Fig. 1) and $l_{\mathrm{p}}=l\left(C_{\infty}+1\right) / 2$ [1], the persistence length, $l_{\mathrm{p}}$, of the model is given by $l_{\mathrm{p}} \simeq 3.38$.

The shape of a polymer is not spherical. A good indicator of its geometrical form is the gyration tensor $\mathrm{Q}$ whose elements are given by 42,44

$$
Q_{\alpha \beta}=\frac{1}{N} \sum_{n=1}^{N}\left(R_{\alpha, n}-R_{\mathrm{cm}, \alpha}\right)\left(R_{\beta, n}-R_{\mathrm{cm}, \beta}\right) \quad(\alpha, \beta=1, \ldots, 3) .
$$

Here, $R_{\alpha, n}$ and $R_{\mathrm{cm}, \alpha}$ denote the $\alpha$ th-component of the position vectors to monomer $n$ and to the chain's center of mass, i.e., $\boldsymbol{R}_{n}=\left(R_{1, n}, R_{2, n}, R_{3, n}\right)$ and $\boldsymbol{R}_{\mathrm{cm}}=\left(R_{\mathrm{cm}, 1}, R_{\mathrm{cm}, 2}, R_{\mathrm{cm}, 3}\right)$. The trace of this tensor provides another way to calculate the radius of gyration 42

$$
\langle\operatorname{Tr} \mathrm{Q}\rangle=\left\langle\lambda_{1}\right\rangle+\left\langle\lambda_{2}\right\rangle+\left\langle\lambda_{3}\right\rangle=R_{\mathrm{g}}^{2},
$$

where $\lambda_{\alpha}$ are the eigenvalues of $\mathrm{Q}$. If the shape of a polymer was spherical, one would have $\left\langle\lambda_{1}\right\rangle=\left\langle\lambda_{2}\right\rangle=\left\langle\lambda_{3}\right\rangle$. Contrary to that, Fig. 目 shows that the biggest eigenvalue, $\left\langle\lambda_{1}\right\rangle$, is much larger than the other two eigenvalues. This implies that the shape of a chain is distorted with respect to a sphere: It is stretched in direction of the long principal axis and shrunk in directions of the axes corresponding to $\left\langle\lambda_{2}\right\rangle$ and $\left\langle\lambda_{3}\right\rangle$. Therefore, a chain rather resembles a "flattened American football"円. This characteristic property can be exploited to construct an efficient coarse-grained model for dense polymer melts [47,48].

In the course of the simulation, a monomer and a lattice direction (out of 6 ) are selected at random, and a jump by one lattice constant is attempted in that direction. The jump is accepted if the resulting bond vectors belong to the allowed set of bonds and if the targeted lattice sites are empty. Otherwise, the move is rejected. This random displacement simulates the impact of a stochastic force which is exerted on a monomer by its local environment.

In the single chain limit, this local jump model is expected to give rise to Rouse dynamics if hydrodynamic interactions are absent [4]. Since hydrodynamic forces are mediated by solvent molecules, an isolated chain of the bond-fluctuation model provides an example for this conjecture [12,36]. However, if the volume fraction $\phi$ increases, deviations from Rouse dynamics are observed due to the mutual interaction of the chains and due to the onset of entanglements [12]. Nevertheless, the Rouse model is generally believed to provide a reliable description of short (non-entangled) chains in a melt. Therefore, we determined its basic variables, the Rouse modes [49]

$$
\boldsymbol{X}_{p}(t)=\frac{1}{N} \sum_{n=1}^{N} \boldsymbol{R}_{n}(t) \cos \frac{(n-1 / 2) p \pi}{N} \quad(p=0,1, \ldots, N-1)
$$

\footnotetext{
${ }^{1}$ Recent studies [45,46] of the shape of Gaussian chains indicate that the visualization of a randomwalk polymer as a flattened American football is not completely correct. The density distribution of monomers in the coordinate system of the principal axes exhibits a slight minimum at the origin (i.e., at the center of mass) for the largest axis, whereas it has a maximum for the other two axes. Therefore, the shape is rather dumbbell-like.
} 
where $\boldsymbol{R}_{n}(t)$ is the position of the $n$th monomer at time $t$. At $t=0$ the (static) correlation of the modes reflects the structural properties of the polymers. For ideal random walk chains it is given by 49

$$
\left\langle\boldsymbol{X}_{p}(0) \cdot \boldsymbol{X}_{q}(0)\right\rangle=\delta_{p q} \frac{b^{2}}{8 N}\left[\frac{1}{\sin (p \pi / 2 N)}\right]^{2} \stackrel{p / N \ll 1}{\longrightarrow} \delta_{p q} \frac{1}{2 \pi^{2}} \frac{N b^{2}}{p^{2}} \quad(\text { for } p>0) .
$$

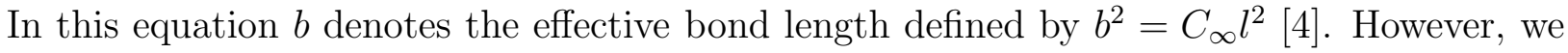
use $b^{2}=R_{\mathrm{e}}^{2} /(N-1)$. This ratio is (slightly) chain-length dependent. It varies from about 8.7 for $N=16$ to about 10.6 for $N=512$, i.e., by about $18 \%$, in our simulation.

Using this identification Eq. (田) suggests that a plot of $8 N(N-1)\left\langle\boldsymbol{X}_{p}(0)^{2}\right\rangle / R_{\mathrm{e}}^{2}$ versus $p / N$ should yield a master curve. Figure 3 shows a test of this prediction. In fact, the data for all chain lengths collapse onto a common curve which is nicely described by $[\sin (p \pi / 2 N)]^{-2}$ if $p / N \lesssim 0.05$. However, if $p / N$ increases, deviations between the simulation results and the Rouse prediction gradually develop. The Rouse theory overestimates the correlation, especially for $p / N \gtrsim 0.3$. These large modes probe local distances along the backbone of a chain. For instance, $p / N \gtrsim 0.3$ corresponds to subunits of a trimer and smaller than that. On these local scales, the intrinsic stiffness of a polymer should be taken into account.

The simplest way to achieve this consists in replacing the random walk by a freely rotating chain [4,41]. This is a chain model, in which the mean bond length and bond angle are fixed and each bond is allowed to rotate freely around the direction of the preceding bond. Starting from

$$
\left\langle\boldsymbol{X}_{p}^{2}(0)\right\rangle=\frac{1}{N^{2}} \sum_{n, m=1}^{N}\left\langle\boldsymbol{R}_{n}(0) \cdot \boldsymbol{R}_{m}(0)\right\rangle \cos \frac{p \pi(n-1 / 2)}{N} \cos \frac{p \pi(m-1 / 2)}{N},
$$

this implies to write (remember $\alpha=\langle\cos \theta\rangle=-0.1055$ )

$$
\left\langle\boldsymbol{R}_{n} \cdot \boldsymbol{R}_{m}\right\rangle=\sum_{i=1}^{n-1} \sum_{j=1}^{m-1}\left\langle\boldsymbol{l}_{i} \cdot \boldsymbol{l}_{j}\right\rangle=l^{2} \sum_{i=1}^{n-1} \sum_{j=1}^{m-1}(-\alpha)^{|i-j|}
$$

instead of $\left\langle\boldsymbol{l}_{i} \cdot \boldsymbol{l}_{j}\right\rangle=l^{2} \delta_{i j}$ for a random walk. Using furthermore

$$
\begin{aligned}
& \sum_{n, m=2}^{N} \cos \frac{p \pi(n-1 / 2)}{N} \cos \frac{p \pi(m-1 / 2)}{N} \sum_{i=1}^{n-1} \sum_{j=1}^{m-1}(-\alpha)^{|i-j|} \\
& =\sum_{n=2}^{N} \sum_{i=2}^{n} \sum_{m=i}^{N} \cos \frac{p \pi(n-1 / 2)}{N} \cos \frac{p \pi(m-1 / 2)}{N}\left[\frac{(-\alpha)^{m-i+1}-1}{(-\alpha)-1}+\frac{(-\alpha)^{n-i+1}-1}{(-\alpha)-1}-1\right],
\end{aligned}
$$

one obtains

$$
\begin{aligned}
\frac{8 N(N-1)}{R_{\mathrm{e}}^{2}}\left\langle\boldsymbol{X}_{p}^{2}(0)\right\rangle \simeq & {\left[\frac{1}{\sin (p \pi / 2 N)}\right]^{2}-\frac{4(-\alpha)}{1-2(-\alpha) \cos (p \pi / N)+(-\alpha)^{2}} \times } \\
& {\left[1+\frac{1}{N} \frac{2 \alpha(1+\alpha)}{1-\alpha} \frac{1}{1+2 \alpha \cos (p \pi / N)+\alpha^{2}} \frac{\sin (p \pi / N)}{\tan (p \pi / 2 N)}\right] }
\end{aligned}
$$


where the $\simeq$-sign indicates that $|\alpha|=0.1055 \ll 1$ was used to write the $1 / N$-correction in the second line of Eq. (5) in the present form. Although this correction violates the $p / N$ scaling, its numerical value is always smaller than 0.03 (= "worst case" for $p=1$ and $N=16$ ) and thus negligible. Therefore, we compare the first line of Eq. (5) to the simulation data. Figure 3 shows that the approximate consideration of stiffness by Eq. (5) reasonably accounts for the suppression of correlations below the Rouse prediction (田). Similar approaches are pursued in [50,51] and tested against experiments and simulations [52,53].

Figure 1 tests another prediction of Eq. (4), the orthogonality of the Rouse modes, i.e., $\left\langle\boldsymbol{X}_{p}(0) \boldsymbol{X}_{q}(0)\right\rangle \sim \delta_{p q}$, for two chain lengths $N=16$ and $N=128$. Whereas the autocorrelation of the first mode is 1 to 2 orders of magnitude larger than cross-correlations with $p>1$ for both chain lengths, this difference in magnitude is only preserved for the

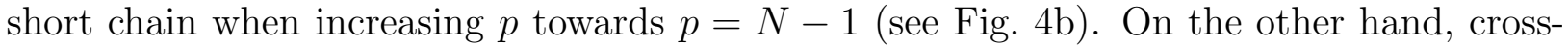
correlations of the same amplitude as the self-correlation develop between $p=127$ and the smallest modes for $N=128$. If one interpretes a difference of 1 to 2 orders of magnitude between self- and cross-correlations as a numerical realization of $\left\langle\boldsymbol{X}_{p}(0) \boldsymbol{X}_{q}(0)\right\rangle \sim \delta_{p q}$, the full spectrum of Rouse modes is only delta-correlated for $N=16$, while small and large modes interfere for large chain lengths.

\section{DYNAMIC PROPERTIES OF THE MELT}

This section discusses the simulation results for dynamic properties of the polymer melt. It is split into three subsections. The first deals with an analysis of various mean-square displacements. The presentation of the data is very much influenced by a recent thorough theoretical and numerical study of the Evans-Edwards model [30-33]. The second subsection

presents the relaxation behavior of the correlation function of the bond-vector, of the end-toend distance and of the Rouse modes, whereas the last subsection discusses the chain-length dependence of the corresponding relaxation times and of the diffusion coefficient.

\section{A. Mean-Square Displacements}

Important quantities to study the crossover from Rouse to reptation dynamics are the following mean-square displacements:

$$
\begin{aligned}
& g_{1}(t)=\left\langle\left[\boldsymbol{R}_{N / 2}(t)-\boldsymbol{R}_{N / 2}(0)\right]^{2}\right\rangle, \\
& g_{2}(t)=\left\langle\left[\boldsymbol{R}_{N / 2}(t)-\boldsymbol{R}_{N / 2}(0)-\boldsymbol{R}_{\mathrm{cm}}(t)+\boldsymbol{R}_{\mathrm{cm}}(0)\right]^{2}\right\rangle, \\
& g_{3}(t)=\left\langle\left[\boldsymbol{R}_{\mathrm{cm}}(t)-\boldsymbol{R}_{\mathrm{cm}}(0)\right]^{2}\right\rangle, \\
& g_{4}(t)=\frac{1}{2}\left(\left\langle\left[\boldsymbol{R}_{1}(t)-\boldsymbol{R}_{1}(0)\right]^{2}\right\rangle+\left\langle\left[\boldsymbol{R}_{N}(t)-\boldsymbol{R}_{N}(0)\right]^{2}\right\rangle\right) .
\end{aligned}
$$

Here, $g_{1}$ is the mean-square displacement of the inner monomer of a chain (situated at position $\boldsymbol{R}_{N / 2}(t)$ at time $\left.t\right), g_{2}$ is the same displacement measured relative to the motion of the chain's center of mass $\left(\boldsymbol{R}_{\mathrm{cm}}(t)\right.$ is position of the center of mass at time $\left.t\right), g_{3}(t)$ is 
the mean-square displacement of the center of mass, and $g_{4}$ is that of the end monomers. Within the framework of reptation theory one expects [3, 14,11, 12]

$$
g_{1}(t) \sim\left\{\begin{array} { l l } 
{ t ^ { 1 / 2 } } & { \text { if } t \ll \tau _ { \mathrm { e } } , } \\
{ t ^ { 1 / 4 } } & { \text { if } \tau _ { \mathrm { e } } \ll t \ll \tau _ { \mathrm { R } } , } \\
{ ( t / N ) ^ { 1 / 2 } } & { \text { if } \tau _ { \mathrm { R } } \ll t \ll \tau _ { \mathrm { d } } , } \\
{ t / N ^ { 2 } } & { \text { if } \tau _ { \mathrm { d } } \ll t , }
\end{array} \quad g _ { 3 } ( t ) \sim \left\{\begin{array}{ll}
t / N & \text { if } t \ll \tau_{\mathrm{e}}, \\
\left(t / N^{2}\right)^{1 / 2} & \text { if } \tau_{\mathrm{e}} \ll t \ll \tau_{\mathrm{R}}, \\
t / N^{2} & \text { if } \tau_{\mathrm{R}} \ll t .
\end{array}\right.\right.
$$

At early times, a chain does not feel the entanglement constraints imposed by its neighbors. Assuming the Rouse model to provide a realistic description for non-entangled chains, one expects $g_{1} \sim t^{1 / 2}$ and $g_{3} \sim t$ in this case. If $t$ equals the entanglement time $\tau_{\mathrm{e}}$, the constraints begin to dominate the polymer dynamics. For larger times, a chain moves as if it were confined in a tube created by its neighbors: It can only slide along the tube axis (the socalled "primitive path"), whereas motion perpendicular to it is suppressed. Since the tube can be thought of as an envelope around the chain's random-walk-like configuration, the chain performs Rouse dynamics along a random walk. This leads to $g_{1} \sim t^{1 / 4}$ and $g_{3} \sim t^{1 / 2}$. If $t$ reaches the Rouse time $\tau_{\mathrm{R}}$, the chain configuration is relaxed inside the tube. For larger times, the center of mass diffuses freely and the inner monomer begins to diffuse out of the tube. The monomer leaves the tube completely if $t$ equals the disentanglement time $\tau_{\mathrm{d}}$.

The power laws of Eq. (7) represent the asymptotic behavior. They can be observed clearly only for very large $N$, where the time scales $\tau_{\mathrm{e}}, \tau_{\mathrm{R}}$ and $\tau_{\mathrm{d}}$ are well separated. For smaller $N$ significant corrections must be expected. This has recently been demonstrated by a detailed theoretical analysis [31] of the Evans-Edwards model [29] and by comparing the analytical results with the outcome of simulations [30,32]. The Evans-Edwards model is a lattice model, in which a single chain moves on a simple cubic lattice through impenetrable obstacles placed in the centers of each unit cell of the lattice (a monomer occupies one lattice site). These obstacles impose a strong geometric confinement on the motion of the chain: The tube diameter $d_{\mathrm{T}}$ is of the order of the lattice constant. Even under these very favorable conditions long chains and long times are required to display the asymptotic power laws clearly [30,32. If one wants to analyze the motion of the inner monomer in a different model, the experience with the studies of [30 33] suggests to use the following three quantities: the mean-square displacement $g_{2}$ which behaves as

$$
g_{2}(t)\left\{\begin{array}{l}
\sim g_{1}(t) \text { if } t \ll \tau_{\mathrm{d}} \\
\rightarrow R_{\mathrm{g}}^{2} \quad \text { if } \quad \tau_{\mathrm{d}} \ll t
\end{array}\right.
$$

so that a transition from $g_{2} \sim t^{1 / 4}$ to $g_{2} \sim t^{1 / 2}$ cannot be interpreted as a crossover to free diffusion due to the saturation of $g_{2}$ at late times, the "cubic invariant" [30 33]

$$
g_{6}(t)=\sqrt{\left\langle\sum_{\alpha=1}^{3}\left[R_{\alpha, N / 2}(t)-R_{\alpha, N / 2}(0)\right]^{4}\right\rangle} \sim g_{1}(t)
$$

which is predicted to be much less plagued than $g_{1}$ by preasymptotic corrections (for the Evans-Edwards model), and the ratio $g_{4}(t) / g_{1}(t)$. This ratio should start around 1 at early times, develop a maximum at intermediate times and approach 1 if $t \rightarrow \infty(=$ free diffusive 
limit for both $g_{4}$ and $g_{1}$ ). For Rouse dynamics the maximum occurs at $t \lesssim \tau_{\mathrm{R}}$ and has the value $g_{4} / g_{1}=2$. If the chain reptates, the analysis of the Evans-Edwards model suggests that the position of the maximum is still given by $t \approx \tau_{\mathrm{R}}$, but its amplitude should be much larger than $2\left(g_{4} / g_{1} \approx 4 \sqrt{2}\right.$ for $\left.N \rightarrow \infty\right)$ [31 33].

Figures 5 and 6 depict the time dependence of $g_{6}$ and $g_{2}$, respectively. The ordinates are divided by $t^{1 / 4}$ to highlight the expected asymptote for the onset of reptation. The figures show that the initial increase of $g_{6}$ and $g_{2}$, which is roughly compatible with a $t^{1 / 2}$-behavior for $200 \lesssim t \lesssim 5000$, considerably slows down when $t>10^{5}$. However, an indication of the $t^{1 / 4}$-power law is only visible for $N=512$. Even for this chain length there is no clear sign of a subsequent $t^{1 / 2}$-increase, but only a fairly protracted transition to the large-time limits $g_{6} \sim t$ and $g_{2} \rightarrow R_{\mathrm{g}}^{2}$. All other chain lengths already cross over to these limits for $t>10^{5}$ (this is especially well visible for $g_{2}$ ). Therefore, much larger (or stiffer [36,54,55]) chains and still better statistics are needed to clearly separate the different power laws and to eventually distinguish between reptation theory and alternative approaches, such as polymer mode-coupling theory [21 26].

Nonetheless, estimates of the tube diameter $d_{\mathrm{T}}$ and the entanglement time $\tau_{\mathrm{e}}$ can be obtained from the figures by posing

$$
g_{6}\left(\tau_{\mathrm{e}}\right) \simeq g_{2}\left(\tau_{\mathrm{e}}\right) \simeq g_{1}\left(\tau_{\mathrm{e}}\right)=\frac{d_{\mathrm{T}}^{2}}{3},
$$

where $\tau_{\mathrm{e}}$ is defined by the intersection point of the $t^{1 / 2}$ - and $t^{1 / 4}$-power laws. The factor $1 / 3$ in Eq. (10) was proposed in [56.57] and justified by the following argument: The tube diameter can be interpreted as the end-to-end distance of a chain segment with $N_{\mathrm{e}}$ monomers. This is the largest segment which does not feel the entanglement constraint yet. It relaxes as a Rouse chain. So, $\tau_{\mathrm{e}}=\tau_{\mathrm{R}}\left(N_{\mathrm{e}}\right)$ which gives $g_{1}\left(\tau_{\mathrm{e}}\right) \approx d_{\mathrm{T}}^{2} / 3$ [ [i]. Qualitatively, the factor $1 / 3$ also appears reasonable. If we assume that the end-to-end vector $\boldsymbol{d}_{\mathrm{T}}^{2}$ is predominantly oriented along the tube axis, the average extension of the tube perpendicular to its axis should be proportional to the radius of gyration $d_{\mathrm{T}}^{2} / 6$ and thus smaller than $d_{\mathrm{T}}^{2}$ [33].

If one accepts these arguments, Eq. (10) yields $d_{\mathrm{T}} \approx 17.9,19.3$ and $\tau_{\mathrm{e}} \approx 84053,100175$ for $g_{2}$ and $g_{6}$, respectively, so that on average $d_{\mathrm{T}} \approx 18.6$ and $\tau_{\mathrm{e}} \approx 92114$. Using the result for $d_{\mathrm{T}}$ we can furthermore estimate the entanglement length $N_{\mathrm{e}}$ by requiring $d_{\mathrm{T}}^{2}=R_{\mathrm{e}}^{2}\left(N_{\mathrm{e}}\right)$. To this end, we fitted the simulation data for $R_{\mathrm{e}}^{2}$ in the range $16 \leq N \leq 64$ by a power law, which gives $R_{\mathrm{e}}^{2}(N)=6.872 N^{1.08}$ so that $N_{\mathrm{e}} \approx 38$. This value is about $26 \%$ larger than the original estimate [34], but coincides very well with the result of a recent study [36].

Figure 7 shows the simulation results for the ratio of the mean-square displacement of the end monomer and the inner monomer, $g_{4}(t) / g_{1}(t)$. The ratio starts around 1.5 at early times, exhibits a maximum at intermediate times and tends to 1 for large $t$. For $N \leq 64$ the maximum occurs close to $g_{4} / g_{1}=2$, i.e., to the value expected from the Rouse model, whereas it is larger for $N=128\left(g_{4} / g_{1} \approx 3\right)$. An increase of the maximum beyond the Rouse limit with growing chain length is predicted theoretically for the EvansEdwards model and proposed as a sensible indicator of reptation dynamics [31 33]. For a reptating chain the disparity between $g_{4}$ and $g_{1}$ has to increase because the motion of the inner monomer is strongly confined by the tube at intermediate times, whereas end monomers always take part in tube renewal. Our data for $N=128$ are indicative of such an enhancement beyond the Rouse limit and are qualitatively similar to simulation results 
of the Evans-Edwards model with a randomly dilute obstacle lattice (see Fig. 5 of [33]). Unfortunately, we have to restrict the discussion to $N \leq 128$ because the calculation of the ratio makes the statistical inaccuracies become so pronounced that a quantitative analysis for $N=512$ was not possible.

In addition to the displacement of inner and end monomers the motion of the center of mass is also interesting. Figure 8 plots $g_{3}(t) / t^{3 / 4}$ versus time for all chain lengths studied. This representation of the ordinate was motivated by Eq. (71). If a chain reptates, $g_{3}(t) / t^{3 / 4}$ should exhibit a minimum close to $t=\tau_{\mathrm{R}}$, which is preceded by a $t^{-1 / 4}$-descent and followed by a $t^{1 / 4}$-ascent. In analogy to $g_{2}$ and $g_{6}$, a signature of this behavior emerges for $N=512$ only, whereas the transition to free diffusion intervenes for smaller chain lengths.

The data reveal that $g_{3}$ is subdiffusive at early times. Particularly for $N=512$, where $\tau_{\mathrm{e}}$ and the crossover-time to free diffusion are well separated, this behavior is very pronounced. The mean-square displacement increases as $g_{3} \sim t^{x}$ with an effective exponent $x \approx 0.84$. Subdiffusive motion of the center of mass at early times is not unusual. It is generally found in computer simulations of linear polymers (see [11, 12] for reviews and [58] for a chemically realistic model of polyethylene) and of rings [59], and it has also been observed lately in experiments (on short chains) [53]. The subdiffusive behavior should be considered as a condensed-phase effect. In a dense melt, a polymer is intermingled with many other chains. Their presence impedes the motion of the center of mass of the tagged chain, leading to subdiffusive behavior. Evidence for this interpretation is provided, for instance, by simulations at different densities. If the density decreases towards the single-chain limit, the bond-fluctuation model yields $g_{3} \sim t$ for all times (i.e., typical Rouse behavior) 12, 34, 36]. Another evidence stems from recent molecular dynamics simulations of the Kremer-Grest model [60]. In this study, the velocity-autocorrelation function of the center of mass was determined. The correlation function becomes negative at intermediate times and approaches 0 from below. This means that the surrounding polymers exert a force on the tagged chain, which reverses its velocity and tries to push it back to its original position.

Since the subdiffusive motion of $g_{3}$ is already present for short non-entangled chains, it should replace the expectation from the Rouse model $g_{3} \sim t$ for $t<\tau_{\mathrm{e}}$ [see Eq. (7)]). Therefore, one can try to determine $\tau_{\mathrm{e}}$ from the intersection of $g_{3} \sim t^{0.84}$ and $g_{3} \sim t^{1 / 2}$. This yields $\tau_{\mathrm{e}} \approx 268718$, which is about a factor of 2.5 larger than the results derived from $g_{6}$ or $g_{2}$. Nevertheless, if one accepts this value, another estimate of $d_{\mathrm{T}}$ can be obtained by posing

$$
g_{3}\left(\tau_{\mathrm{e}}\right)=\frac{b^{2} d_{\mathrm{T}}^{2}}{3 R_{\mathrm{e}}^{2}}\left(\frac{k_{\mathrm{B}} T}{\zeta b^{2}} \tau_{\mathrm{e}}\right)^{1 / 2},
$$

where the factor $1 / 3$ stems from Eq. (10) and the requirement $g_{1}\left(\tau_{\mathrm{d}}\right)=g_{3}\left(\tau_{\mathrm{d}}\right)$. In Eq. (11), $\zeta$ denotes the monomeric friction coefficient and $\tau_{\mathrm{e}}$ is (assumed to be) given by [12]

$$
\tau_{\mathrm{e}}=\left(\frac{d_{\mathrm{T}}}{b}\right)^{4} \frac{\zeta b^{2}}{k_{\mathrm{B}} T},
$$

so that

$$
d_{\mathrm{T}}=\left[3 g_{3}\left(\tau_{\mathrm{e}}\right) R_{\mathrm{e}}^{2}\right]^{1 / 4} .
$$

Using $g_{3}\left(\tau_{\mathrm{e}}\right) \approx 10.37$ and $R_{\mathrm{e}}^{2}=5348$, Eq. (13) leads to $d_{\mathrm{T}} \approx 20.3$. This value is compatible with previous estimates from $g_{6}$ and $g_{2}$. However, due the small exponent $1 / 4$ in Eq. (13) 
the result for the tube diameter is not very sensible to variations of $\tau_{\mathrm{e}}$. For instance, when using the average $\tau_{\mathrm{e}}$ from $g_{6}$ and $g_{2}$, i.e., $\tau_{\mathrm{e}} \approx 92114$, so that $g_{3}\left(\tau_{\mathrm{e}}\right) \approx 4.27$, one obtains $d_{\mathrm{T}} \approx 16.2$ which is also in reasonable agreement with the results derived from the monomer displacements.

On the other hand, the power law $g_{3} \sim t^{1 / 2}$ provides a further possibility to determine $d_{\mathrm{T}}$ by the intersection with the diffusive behavior $g_{3} \sim t$. The intersection point should define the Rouse time $\tau_{\mathrm{R}}\left(\approx 4.34 \times 10^{6}\right)$, and one expects the value $g_{3}\left(\tau_{\mathrm{R}}\right)$ to be close to $d_{\mathrm{T}}^{2}$. If we determine the prefactor by the same argument which led to Eq. (11), we obtain $g_{3}\left(\tau_{\mathrm{R}}\right)=d_{\mathrm{T}}^{2} / 3$. Using $g_{3}\left(\tau_{\mathrm{R}}\right) \approx 54.8$, we find $d_{\mathrm{T}} \approx 13$. This value seems too small in comparison with the previous results which indicates that the Rouse time is presumably larger than determined. Therefore, $\tau_{\mathrm{R}}=4.34 \times 10^{6}$ rather corresponds to a lower bound than to a reliable estimate.

\section{B. Correlation Functions}

If a chain starts to reptate, the motion of the monomers perpendicular to the primitive path is restricted to displacements smaller than the tube diameter. This implies that the decay of orientational correlation functions of chain segments should significantly slow down if the size of the segment becomes comparable to $d_{\mathrm{T}}$. Therefore, correlation functions which probe reorientations on different length scales along the backbone of a chain are interesting quantities.

The smallest segment along the backbone of a chain is the bond vector. The correlation function of the bond vector can be defined by

$$
\phi_{\mathrm{b}}(t)=\frac{1}{(N-1) l^{2}} \sum_{n=1}^{N-1}\left\langle\boldsymbol{l}_{n}(t) \cdot \boldsymbol{l}_{n}(0)\right\rangle
$$

where $l^{2}=\left\langle\boldsymbol{l}^{2}(0)\right\rangle$ is the mean-square bond length. This quantity is also interesting because it should be closely related to the shear modulus [26]. Figure 9 illustrates the time- and chain-length dependences of $\phi_{\mathrm{b}}(t)$. The data are presented in a scaling plot where the time axis is given in units of the relaxation time, $\tau_{\mathrm{b}}$, of the bond vector defined by $\phi_{\mathrm{b}}\left(\tau_{\mathrm{b}}\right)=1 / \mathrm{e}$. This time scale depends weakly on chain length (see Table $\mathbb{1}$ ). If $\tau_{\mathrm{b}}$ was the only relevant time scale, all $\phi_{\mathrm{b}}(t)$ 's, measured for different $N$, should collapse onto a master curve. Figure 9 shows that such a collapse is realized for about $75 \%$ of the relaxation, whereas the final decay to zero depends on chain length. Larger chains relax more slowly than shorter ones.

Such a behavior is already expected from the (discrete [49]) Rouse model which expresses $\phi_{\mathrm{b}}(t)$ as

$$
\phi_{\mathrm{b}}(t)=\frac{1}{N-1} \sum_{p=1}^{N-1} \phi_{p}(t)=\frac{1}{N-1} \sum_{p=1}^{N-1} \exp \left(-\frac{t p^{2}}{\tau_{\mathrm{R}}}\right)
$$

where $\tau_{\mathrm{R}}$ is the Rouse time and $\phi_{p}(t)$ the correlation function of the $p$ th Rouse mode 4,49

$$
\phi_{p}(t)=\frac{\left\langle\boldsymbol{X}_{p}(t) \cdot \boldsymbol{X}_{p}(0)\right\rangle}{\left\langle\boldsymbol{X}_{p}^{2}(0)\right\rangle}=\exp \left(-\frac{t p^{2}}{\tau_{\mathrm{R}}}\right) .
$$


If $t \ll \tau_{\mathrm{R}}$, the relaxation behavior of the sum in Eq. (15) is dominated by large $p$ 's, which yields $\phi_{\mathrm{b}}(t) \sim 1 / t^{1 / 2}$ independent of $N$ (in the continuum limit). On the other hand, if $t \gtrsim \tau_{\mathrm{R}}, \phi_{\mathrm{b}}(t)$ relaxes the more slowly, the longer the chain length. These predictions seem to be in accord with the simulation results. However, there are differences at short times for all chain lengths and at long times for large $N$.

At short times, the Rouse formula (15) underestimates the relaxation strength of $\phi_{\mathrm{b}}(t)$. This discrepancy is caused by Eq. (16) which assumes an exponential decay for all Rouse modes. However, especially the simulation results for large Rouse modes do not decay in a simple exponential fashion (see discussion of Fig. 12). These large modes determine the behavior of $\phi_{\mathrm{b}}(t)$ at short times, whereas the late-time relaxation is dominated by the smallest Rouse mode which is almost exponential for $N \leq 128$. Nevertheless, Eqs. (15) and (16) only provide a reasonable description for the $N$-dependent tail of $\phi_{\mathrm{b}}(t)$ if $N=16$. For larger $N$ the simulation data relax more slowly and finally develop a power-law decay if $N \geq 128$. The power law starts around $t \approx \tau_{\mathrm{e}}$ and extends over about 3 decades up to the end of our simulation. With the present simulation results we cannot decide whether the power law is an indication of a possible two-step relaxation which would become clearly visible for longer chains, as suggested by polymer mode-coupling theory [24,26].

Qualitatively, the slow power-law decay of $\phi_{\mathrm{b}}(t)$ implies that some bonds lose the memory of their original orientation only very gradually. Persistence of bond orientation is also an important notion in the development of the continuum theory of reptation 4 . There, a key quantity is the probability $\psi(s, t)$ that a segment $s$ of the primitive chain is still in the tube at time $t$. This probability is related to the projection of the (unit) tangent vectors, $\boldsymbol{u}\left(s^{\prime}, t\right)$, of all segments $s^{\prime}$ onto one primitive path segment $s$, i.e., onto $\boldsymbol{u}(s, 0)$ (see Eq. (6.45) of Ref. «). In analogy to $\psi(s, t)$, we define

$$
\phi_{n}(t)=\frac{\sum_{n^{\prime}=1}^{N}\left\langle\boldsymbol{l}_{n^{\prime}}(t) \cdot \boldsymbol{l}_{n}(0)\right\rangle}{\sum_{n^{\prime}=1}^{N}\left\langle\boldsymbol{l}_{n^{\prime}}(0) \cdot \boldsymbol{l}_{n}(0)\right\rangle},
$$

which represents the probability that the original orientation of the bond vector $\boldsymbol{l}_{n}(0)$ is still present at time $t$. This quantity can be calculated by the (discrete 49]) Rouse model. The result is

$$
\begin{aligned}
& \phi_{n}(t)=\frac{\sum_{p=1 \text { (odd) }}^{N-1}[\sin (n p \pi / N) / \tan (p \pi / 2 N)] \exp \left(-p^{2} t / \tau_{\mathrm{R}}\right)}{\sum_{p=1(\text { odd })}^{N-1} \sin (n p \pi / N) / \tan (p \pi / 2 N)} \\
& \stackrel{p / N \ll 1}{=} \frac{4}{\pi} \sum_{\substack{p=1 \\
(p \text { odd })}}^{\infty} \frac{1}{p} \sin (n p \pi / N) \exp \left(-p^{2} t / \tau_{\mathrm{R}}\right) .
\end{aligned}
$$

The continuum limit (19) is identical to the reptation formula for $\psi(s, t)$ if $\tau_{\mathrm{R}}$ is replaced by the disentanglement time $\tau_{\mathrm{d}}$. Numerically, the discrete and continuum results for $\phi_{n}(t)$ are almost indistinguishable. Therefore, we compare Eq. (18) with simulation data for $N=16$ and $N=128$ in Fig. 10 .

If $N=16$, Eq. (18) provides a fairly accurate description of the simulated $\phi_{n}(t)$. Both theory and simulation yield bell-shaped curves whose amplitudes become smaller as time increases. This behavior can be rationalized as follows: Since chain ends are more mobile than inner monomers, bond vectors close to the ends decorrelate more quickly than those in 
the inner part of the chain. The decorrelation thus propagates from the chain ends towards the middle monomer. The bond vector orientation of the inner monomer is rather longlived. Even at $t=\tau_{\text {ee }}$, where the end-to-end vector correlation has decayed to about $30 \%$ of its original value (see Eq. (21) and Fig. 11), $\phi_{n}(t)$ is still about 0.5 for $n=8$. This slow relaxation is already present for short, Rouse-like chains and should not be considered as a characteristic feature of reptation-like dynamics, since the Rouse and reptation formulas agree with one another [Eq. (19)].

However, the simulation results for $\phi_{n}(t)$ exhibit differences between short and long chains. As Fig. 10 shows, the relaxation of inner monomers becomes strongly retarded compared to the theoretical (reptation) prediction if $N=128$. More than $50 \%$ of the initial orientation of the inner third of the chain is preserved even at $\tau_{\text {ee. }}$ These long-lived correlations, which are not present for small $N$, should be responsible for the power-law behavior of $\phi_{\mathrm{b}}(t)$ (see Fig. 9) because they begin to develop in the same time window as the power law, i.e, for times larger than the entanglement time $\left(\tau_{\mathrm{e}} / \tau_{\mathrm{ee}} \approx 0.04\right.$ for $\left.N=128\right)$, and last until $t>\tau_{\text {ee }}$ (the time $t / \tau_{\mathrm{b}}=10^{4}$ in Fig. 9 corresponds to about $t / \tau_{\text {ee }} \approx 2$ in Fig. 10 b).

Furthermore, they should also become visible in the motion of the monomers, particularly when comparing the mean-square displacement of the end monomer, $g_{4}(t)$, with that of the inner monomer $g_{1}(t)$. Figure 10 suggests that the ratio $g_{4}(t) / g_{1}(t)$ for $t \approx \tau_{\text {ee }}$ is much larger for $N=128$ than for $N=16$. Our simulation data for $g_{4} / g_{1}$ support this expectation (see Fig. (7).

If the chain length increases, one may expect the slowly relaxing zone around the middle monomer to grow and to influence the dynamic behavior of the chain more and more. For instance, one might speculate that the end-to-end vector relaxes in two steps: There is a first step initiated by the chain ends. Their fast motion decorrelates adjacent bond vectors very efficiently. However, this decorrelation does not propagate homogeneously along the polymer backbone towards the inner monomer as it is the case for short chains, but slows down in the inner part of the chain. The relaxation of this part is responsible for the second step.

Some evidence for this conjecture is given in Fig. 11 which shows the time-dependence of the end-to-end vector correlation function, $\phi_{\mathrm{e}}(t)$, for all chain lengths studied and compares the simulation data with the Rouse (or reptation) prediction

$$
\phi_{\mathrm{e}}(t)=\frac{\left\langle\boldsymbol{R}_{\mathrm{e}}(t) \cdot \boldsymbol{R}_{\mathrm{e}}(0)\right\rangle}{\left\langle\boldsymbol{R}_{\mathrm{e}}(0)^{2}\right\rangle}=\sum_{\substack{p=1 \\(p \text { odd })}}^{\infty} \frac{8}{p^{2} \pi^{2}} \exp \left(-p^{2} t / \tau_{\mathrm{ee}}\right)
$$

where the relaxation time $\tau_{\text {ee }}$ is defined by

$$
\phi_{\mathrm{e}}\left(\tau_{\mathrm{ee}}\right)=\sum_{\substack{p=1 \\(p \text { odd })}}^{\infty} \frac{8}{p^{2} \pi^{2}} \exp \left(-p^{2}\right)=0.298221
$$

Note that Eq. (20) is the same for both Rouse and reptation theory, the only difference being that $\tau_{\text {ee }}$ is the Rouse time, $\tau_{\mathrm{R}}$, in the first case and the disentanglement time, $\tau_{\mathrm{d}}$, in the latter case, i.e., 


$$
\tau_{\mathrm{ee}}= \begin{cases}\tau_{\mathrm{R}}=\frac{1}{3 \pi^{2}} \frac{\zeta b^{2}}{k_{\mathrm{B}} T} N^{2} & \text { if } \quad N \ll N_{\mathrm{e}}, \\ \tau_{\mathrm{d}}=\frac{1}{\pi^{2}} \frac{\zeta b^{2}}{k_{\mathrm{B}} T} \frac{b^{2}}{d_{\mathrm{T}}^{2}} N^{3} \quad \text { if } \quad N \gg N_{\mathrm{e}} .\end{cases}
$$

where prefactors $1 / 3 \pi^{2}$ and $1 / \pi^{2}$ are taken from [4] (they have been set equal to 1 in the discussion of the mean-square displacements in Sect. [IIA).

Equation (20) suggests that simulation data for $\phi_{\mathrm{e}}(t)$ depend on $N$ only via $\tau_{\text {ee }}$. By using a rescaled time axis, $t / \tau_{\text {ee }}$, it should be possible to superimpose all data onto a master curve which is given by Eq. (20). Figure 11 confirms this expectation if $N \leq 128$. For $N=512$, however, the relaxation is more complicated. It seems to occur in two steps. The first step starts at about $t \approx \tau_{\mathrm{e}}$, and the second takes place in the time window where $g_{3}$ crosses over to free diffusion, i.e., on the scale of the Rouse time $\tau_{\mathrm{R}}$ (see Fig. 8). This behavior is similar to that expected from polymer mode-coupling theory [24 26]. The theory predicts two power laws: $1-\phi_{\mathrm{e}}(t) \sim t^{9 / 32}$ for $\tau_{\mathrm{e}} \ll t \ll \tau_{\mathrm{R}}$ and $1-\phi_{\mathrm{e}}(t) \sim t^{3 / 8}$ for $\tau_{\mathrm{R}} \ll t \ll \tau_{\mathrm{RR}}$ $\left(\tau_{\mathrm{RR}}=\right.$ renormalized Rouse time $\left.<\tau_{\mathrm{d}}\right)$. Our data are compatible with a $t^{9 / 32}$-behavior for about one decade $\left(0.002 \lesssim t / \tau_{\text {ee }} \lesssim 0.1\right)$ and eventually with $t^{3 / 8}$ close to $\tau_{\text {ee. }}$ Of course, this can only be considered as an indication that these predictions might be relevant for our model. Clarification of this point requires a much better separation of the relevant time scales and thus simulations of longer chains. On the other hand, it is not obvious that Eqs. (20) and (21) are accurate for chain lengths in the crossover regime from Rouse to reptation behavior, although they describe both limiting cases of the simple Rouse and the "pure" (i.e., asymptotic) reptation theory. Certainly, a quantitatively reliable theoretical prediction for $\phi_{\mathrm{e}}(t)$ as well as for $\tau_{\mathrm{ee}}$ in the regime where $N$ and $N_{\mathrm{e}}$ are comparable would be very desirable.

The observed two-step-like relaxation behavior is not limited to $\phi_{\mathrm{e}}(t)$. It can also be observed for the correlation function of the Rouse modes $\phi_{p}(t)$. Figure 12 shows scaling plots of $\phi_{p}(t)$ for $N=16$ and $N=512$. As suggested by Eq. (16), the scaling time, $\tau_{p}$, is defined by $\phi_{p}\left(\tau_{p}\right)=1 / \mathrm{e}$. The figure illustrates that the first Rouse mode is almost exponential for $N=16$, whereas the relaxation of higher modes becomes progressively nonexponential with increasing $p$ (smaller distance along the chain backbone). However, the curves cannot be described (completely) by a stretched exponential. This would imply that the fast decay of, say, $p=15$ for $\phi_{\mathrm{e}}(t)>1$ /e entails a correspondingly slower relaxation if $\phi_{\mathrm{e}}(t)<1 / \mathrm{e}$. Since the data splay out with increasing $p$ at short times, they should also splay out at late times, but in reverse order. There is no indication of that behavior in Fig. 12a. The interplay of chain stiffness and local excluded volume forces gives rise to a more complicated than stretched-exponential time dependence. The same behavior is also observed for a bead-spring model of short chains [61], and, to some extent, for $N=512$ in Fig. 12b. For large chains, however, there is another factor which may contribute: crosscorrelations between Rouse modes. Figure 3 revealed that these can be as pronounced as the self-correlation if $p=N-1$. Since $\phi_{p=1}(t)$ exhibits again the signature of a two-step relaxation, such as $\phi_{\mathrm{e}}(t)$, this behavior could also influence the decay of $\phi_{p=511}(t)$. 


\section{Correlation Times and Diffusion Coefficient}

The discrete Rouse model yields the following expression for the relaxation time $\tau_{p}$ [49]

$$
\begin{aligned}
\tau_{p} & =\frac{1}{12} \frac{\zeta}{k_{\mathrm{B}} T}\left[\frac{b}{\sin (p \pi / 2 N)}\right]^{2} \\
& =\frac{2}{3} \frac{N \zeta}{k_{\mathrm{B}} T}\left\langle\boldsymbol{X}_{p}^{2}(0)\right\rangle
\end{aligned}
$$

where Eq. (田) was used to obtain the last line. The unit of $\tau_{p}$ is set by the monomeric friction coefficient $\zeta$ which should be independent of the mode index $p$ and chain length $N$. Within the framework of the Rouse model $\zeta$ can be determined from the diffusion coefficient, $D$, of a chain by

$$
N D=\frac{k_{\mathrm{B}} T}{\zeta} .
$$

If one furthermore identifies the effective bond length with $b^{2}=R_{\mathrm{e}}^{2} /(N-1)$ (as already done in Fig. 3), Eq. (23) suggests to construct a master curve by plotting $12 D N(N-1) \tau_{p} / R_{\mathrm{e}}^{2}$ versus $p / N$. Figure 13 shows that such a scaling is only possible if $N \leq 64$. For these chain lengths the scaling yields a curve which is independent of $N$ and coincides with the Rouse prediction for $p / N \lesssim 0.06$. On the other hand, there are deviations for $p / N \geq 0.3$, where Eq. (23) overestimates the simulation results.

Qualitatively, this behavior is identical to that observed for $\left\langle\boldsymbol{X}_{p}^{2}(0)\right\rangle$ in Fig. 3. This suggests that the discrepancy between simulation and Eq. (23) might be related to local stiffness effects and that it could be removed by expressing $\left\langle\boldsymbol{X}_{p}^{2}(0)\right\rangle$ via Eq. (5) or by inserting the simulation results for $\left\langle\boldsymbol{X}_{p}^{2}(0)\right\rangle$ in Eq. (24). The latter choice was made in the inset of Fig. 13. This yields a good description for $N \leq 64$. Two conclusions can be drawn from that: First, as expected from Eq. (24), $\left\langle\boldsymbol{X}_{p}^{2}(0)\right\rangle$ carries the whole $p$-dependence so that $\zeta$ is constant. This should be considered as a property of the bond-fluctuation model and not as a general feature because a bead-spring model can exhibit a different behavior [61|2. Second, although the diffusion coefficients for $N \leq 64$ do not obey Eq. (25) [see Fig. 14], the Rouse relation (24) effectively remains valid. This is possible because the simulation results for $\tau_{p}$ can be written as $\tau_{p}=f(N)[\sin (p \pi / 2 N)]^{-2}$ and the dependence of the function $f$ on $N$ is just given by $\left(N(N-1) D / R_{\mathrm{e}}^{2}\right)^{-1}$. Theoretically, $N(N-1) D / R_{\mathrm{e}}^{2}$ should represent the reorientation time of a monomer, $\zeta b^{2} / k_{\mathrm{B}} T$, which is independent of $N$. In practice, $N(N-1) D / R_{\mathrm{e}}^{2}$ varies from about $6 \times 10^{-4}$ for $N=16$ to about $3.5 \times 10^{-4}$ for $N=64$, i.e., by about $40 \%$. For larger chain length, however, the residual $N$-dependence of $\tau_{p}$ can no longer be lumped in a prefactor, although $N(N-1) D / R_{\mathrm{e}}^{2}$ decreases to about $7 \times 10^{-5}$

\footnotetext{
${ }^{2}$ This point was not stated explicitly in Ref. 61. However, since roughly $\left\langle\boldsymbol{X}_{p}^{2}(0)\right\rangle \sim p^{-2.2}$ and $\tau_{p} \sim p^{-2}$ in this case, Eq. (24) would imply $\zeta=\zeta_{p} \sim p^{0.2}$. Similar observations were also made in neutron scattering experiments on polyisobutylene [52]. A quantitative description of the experimental results was only possible if both local stiffness and an increase of $\zeta$ with increasing mode index $p$ were taken into account.
} 
for $N=512$ so that it is 1 order of magnitude smaller than for $N=16$. Nonetheless, $\tau_{p}$ depends more strongly on $N$ than $N(N-1) D / R_{\mathrm{e}}^{2}$ for small $p$ and does not seem to be any longer a function of $p / N$ alone. Even if one changes the prefactor to shift the data for $N=128$ onto those for $N=512$, one can guess from Fig. 13 that such a collapse is only possible for $p / N \gtrsim 0.03$, whereas $\tau_{p}$ for $N=512$ becomes larger than that for $N=128$ when $p / N<0.03$. Since scaling with $p / N$ was possible for $\left\langle\boldsymbol{X}_{p}^{2}(0)\right\rangle$, which probes the static properties of a chain only, Fig. 13 suggests that the friction coefficient $\zeta$ becomes $p$ - and $N$-dependent.

Finally, Fig. 14 shows the variation of $\tau_{\text {ee }}$ [defined by Eq. (21)] and of $D$ with chain length. The ordinates were scaled by the chain length dependence expected from the Rouse model, i.e., $\tau_{\text {ee }} / N^{2}$ and $N D$ [see Eqs. (22) and (25)]. The figure illustrates that neither $\tau_{\text {ee }}$ nor $D$ behave Rouse-like for small $N$ or reptation-like for large $N$. A possible explanation for this finding is given by a recent reanalysis [36] of the crossover scaling from the semi-dilutesolution to the melt state of the bond-fluctuation model [34]. This new analysis focused on the diffusion coefficient, but substantially varied chain stiffness, which had not been done before. The important outcome for the present work is that the diffusion data of Fig. 14 just lie in the crossover regime between Rouse dynamics, which is realized by flexible chains in dilute solution, and reptation dynamics which is most pronounced for stiff chains in the melt. Apparently, chain stiffness reduces the tube diameter and thereby amplifies the reptation behavior. The same conclusion has also been suggested by a recent molecular-dynamics simulation of the Kremer-Grest model [54]. Since the diffusion data belong to the crossover regime, this should also hold for $\tau_{\text {ee }}$ in Fig. 14 so that neither $\tau_{\text {ee }} \sim N^{2}$ nor $\tau_{\text {ee }} \sim N^{3}$ can be observed. If one just fitted the last two chain lengths $(N=128,512)$ by a power law, one would obtain $\tau_{\text {ee }} \sim N^{3.23}$. This is similar to experiments where one often finds that the relaxation time scales as $N^{3.4}$ [5,6]. Of course, the present simulation data cannot prove, but only provide an indication that the bond-fluctuation model approaches (the possible asymptotic behavior) $\tau_{\text {ee }} \sim N^{3}$ from below with an exponent larger than 3 , as suggested theoretically [4, 16, 31]. These theoretical studies show that the crossover to the reptation asymptote is postponed to very large $N$. To observe this asymptote one has to study either much longer or (eventually) stiffer chains which seem to show a clearer signature of reptation for accessible chain lengths [36,54].

Nevertheless, one can use the simulation results for $\tau_{\text {ee }}$ to estimate the entanglement length $N_{\mathrm{e}}$ by requiring that $\tau_{\mathrm{e}}$ corresponds to the relaxation time of a chain with end-to-end vector $d_{\mathrm{T}}^{2}=R_{\mathrm{e}}^{2}\left(N_{\mathrm{e}}\right)$. To read off $N_{\mathrm{e}}$, we fitted $\tau_{\mathrm{ee}}$ in the range of $\tau_{\mathrm{e}}(=92114)$, i.e., for $16 \leq N \leq 64$, by a power law. This gives $\tau_{\mathrm{ee}}=24.71 N^{2.31}$ so that $N_{\mathrm{e}} \approx 35$. This value is compatible with our previous estimate from $d_{\mathrm{T}}^{2}=R_{\mathrm{e}}^{2}\left(N_{\mathrm{e}}\right)$, i.e., $N_{\mathrm{e}} \approx 38$ (see Sect. ПIA), so that on average $N_{\mathrm{e}} \approx 37$. This result agree very well with that obtained by the above mentioned reanalysis of the crossover scaling [36].

\section{CONCLUSIONS}

The present paper summarizes an attempt to extend previous work [12, 34, 35] on the dynamics of long chain polymer melts towards larger chain lengths and longer times. The aim was to sufficiently enter the reptation regime to perform a careful test of the theory. However, it turned out that the crossover from non-entangled to entangled dynamics is 
very gradual. Even the longest chain, $N=512$, which is about 13 times larger than the entanglement length $N_{\mathrm{e}}$, can only be considered as slightly entangled when analyzed in terms of the asymptotic power laws of reptation theory. This finding is expected from a theoretical analysis of the Evans-Edwards model [30 33] and molecular dynamics simulations [56.57].

For $N=512$ we find evidence that there is an entanglement time $\tau_{\mathrm{e}}$, above which the mean-square displacements of inner monomers, $g_{1}(t)\left[\approx g_{6}(t)\right]$ and $g_{2}(t)$, and that of the center of mass, $g_{3}(t)$, increase as $\sim t^{1 / 4}$ and $\sim t^{1 / 2}$, respectively. From these quantities the tube diameter $d_{\mathrm{T}}$ and $\tau_{\mathrm{e}}$ can be estimated: $d_{\mathrm{T}} \approx 18.6$ and $\tau_{\mathrm{e}} \approx 92114$. Using these values and requiring $R_{\mathrm{e}}^{2}\left(N_{\mathrm{e}}\right)=d_{\mathrm{T}}^{2}$ and $\tau_{\mathrm{ee}}\left(N_{\mathrm{e}}\right)=\tau_{\mathrm{e}}$, the entanglement length can be read off of the simulation data: $N_{\mathrm{e}} \approx 37$. The quoted values for $d_{\mathrm{T}}$ and $\tau_{\mathrm{e}}$ are averages derived from $g_{2}$ and $g_{6}$. The results from $g_{2}$ and $g_{6}$ were rather close, whereas those from $g_{3}$ sometimes deviate substantially and thus appear less reliable (perhaps due to preasymptotic effects, as pointed out in [33]). Of course, there are still uncertainties connected with these numbers, especially with $d_{\mathrm{T}}$, since the prefactors are unknown and $N=512$ is not really asymptotic. Here, the important point is that they are consistent with one another and with other independent studies. For instance, the value $N_{\mathrm{e}} \approx 37$ agrees very well with that found in [36].

In addition to mean-square displacements evidence for reptation-like dynamics can be found in orientational correlation functions. Bond vectors in the innermost part of a chain decorrelate from their initial orientations more slowly than bonds close to the ends. This difference is present for all chain lengths, but becomes much more pronounced if $N \gg N_{\mathrm{e}}$ and $\tau_{\mathrm{e}} \ll t \ll \tau_{\mathrm{ee}}$. If there was a confining tube around a chain, such a behavior would be expected because reorientations of inner monomers are strongly hindered as long as the geometric constraints exist (i.e., for $t<\tau_{\mathrm{ee}}$ ), whereas chain ends always take part in tube renewal and thus dissolve the confinement immediately. The simulations suggest that sensible indicators of this behavior are a late-time power law decay of the bond-vector correlation function and a modulated (eventually two-step) relaxation of the end-to-end vector or Rouse modes correlation functions as well as the ratio $g_{4}(t) / g_{1}(t)$, as proposed in [31 33]. The challenging problem consists in determining these functions with sufficient statistical accuracy in the relevant range of chain lengths and times.

For short chains $\left(N \lesssim N_{\mathrm{e}}\right)$ the Rouse model represents a viable, though not perfect approach. Deviations are observed particularly on short length scales along the polymer backbone. This is to be expected because the microscopic potentials of the polymer should strongly influence the local dynamics. In general, these potentials cannot be represented by a flexible concatenation of Rousian harmonic springs, but introduce some local stiffness. If stiffness is taken into account, one can obtain a (almost) quantitative description of the static correlation $\left\langle\boldsymbol{X}_{p}^{2}(0)\right\rangle$ and of the $p$-dependence of the Rouse modes' correlation time $\tau_{p}$, which scales as $\tau_{p} \sim\left\langle\boldsymbol{X}_{p}^{2}(0)\right\rangle$. Whereas the former property could be a general feature, the scaling $\tau_{p} \sim\left\langle\boldsymbol{X}_{p}^{2}(0)\right\rangle$ is certainly a property of our model. Simulations of other models [61] and neutron scattering experiments on polyisobutylene [52] suggest that the monomeric friction coefficient can be mode dependent. Such findings are plausible because one would in general not expect that static properties can uniquely determine the dynamic behavior.

Another characteristic dynamic property of the Rouse modes is the progressive nonexponential character of the relaxation with increasing mode index $p$. This behavior is not a special feature of the bond-fluctuation model, but is also observed for a bead-spring model [61] and in chemical realistic simulations of polyethylene [58]. These deviations from a pure 
exponential decay are important if one wants to calculate the relaxation of quantities, for which large modes and short times also contribute significantly. An example is provided by the bond-vector correlation function (see Fig. 9). If one inserts the simulated Rouse modes instead of Eq. (16) into Eq. (15), a perfect description of the correlation function is obtained. Since this description only requires the orthogonality of the Rouse modes, it shows that the melt dynamics does not introduce (significant) correlations between different Rouse modes, i.e., $\left\langle\boldsymbol{X}_{p}(t) \cdot \boldsymbol{X}_{q}(0)\right\rangle \propto \delta_{p q}$. We believe that this property is general, since it is also observed for other models 61.

A further important feature is that there is no significant regime of chain lengths where the Rouse prediction $D \sim 1 / N$ holds (see inset of Fig. 14). Furthermore, the related prediction $g_{3}(t) \sim t$ (for $t \gtrsim 1$ in our units) is also not valid, not even for $N=16$ (see Fig. 8). If $g_{3}(t) \sim t$ were true, the log-log plot $g_{3} / t^{3 / 4}$ versus $t$ should exhibit a straight line with slope $1 / 4$. However, we find a curved behavior spread over several decades. Our results (Fig. 14) imply that there are about two decades in chain length $\left(10 \leq N \leq 10^{3}\right)$ which are neither fully described by the simple Rouse model nor by asymptotic reptation theory. A more complete theoretical description is thus called for.

\section{ACKNOWLEDGEMENT}

We are indebted to J.-L. Barrat, R. Everaers, F. Eurich, M. Fuchs, P. Maass, C. Mischler, W. Paul, J. Wittmer for helpful discussions on various aspects of this work. This study would not have been possible without a generous grant of simulation time by the HLRZ Jülich, the RHRK Kaiserslautern and the computer center at the University of Mainz. Financial support by the ESF Programme on "Experimental and Theoretical Investigation of Complex Polymer Structures" (SUPERNET) is gratefully acknowledged. 


\section{REFERENCES}

[1] P.G. de Gennes, J. Chem. Phys. 55, 572 (1971).

[2] P.G. de Gennes, J. Physique 42, 735 (1981).

[3] P.G. de Gennes, Scaling Concepts in Polymer Physics (Cornell University Press, Ithaca, 1979).

[4] M. Doi, S.F. Edwards, The Theory of Polymer Dynamics (Oxford University Press, New York, 1986).

[5] W.W. Graessley, Adv. Polym. Sci. 47, 68 (1982).

[6] T.P. Lodge, N.A. Rotstein, S.Prager, Adv. Chem. Phys. 79, 1 (1990).

[7] B. Ewen, D. Richter, Adv. Polym. Sci. 134, 1 (1997).

[8] S. Pahl, G. Fleischer, F. Fujara, B. Geil, Macromolecules 30, 1414 (1997).

[9] K.A. Welp, R.P. Wool, S.K. Satija, S. Pispas, J. Mays, Macromolecules 31, 4915 (1998).

[10] A. Baumgärtner, Ann. Rev. Phys. 35, 419 (1984).

[11] K. Kremer, G.S. Grest, Entanglement Effects in Polymer Melts and Networks. In: Monte Carlo and Molecular Dynamics Simulations in Polymer Science, edited by K. Binder (Oxford University Press, New York, 1995), pp. 194-271.

[12] K. Binder, W. Paul, J. Polym. Sci. B 35, 1 (1997).

[13] A.N. Semenov, M. Rubinstein, Eur. Phys. J B 1, 87 (1998).

[14] M. Doi, J. Polym. Sci. 21, 667 (1983).

[15] S.T. Milner, T.C.B. McLeish, Phys. Rev. Lett. 81, 725 (1998).

[16] T.C.B. McLeish, S.T. Milner, Adv. Poly. Sci. 143, 195 (1999).

[17] P.G. de Gennes, Macromolecules 9, 587 (1976); ibid., 594 (1976).

[18] M. Daoud, P.G. de Gennes, J. Polym. Sci. Phys. ed. 17, 1971 (1979).

[19] M. Rubinstein, S.P. Obukhov, Phys. Rev. Lett. 71, 1856 (1993).

[20] A.N. Semenov, Physica A 166, 263 (1990).

[21] K.S. Schweizer, J. Chem. Phys. 91, 5802 (1989); ibid., 5822 (1989).

[22] K.S. Schweizer, Physica Scripta 49, 99 (1993).

[23] K.S. Schweizer, G. Szamel, Trans. Theo. Stat. Phys. 24, 947 (1995).

[24] K.S. Schweizer, M. Fuchs, G. Szamel, M. Guenza, H. Tang, Macromol. Theory Simul. 6, 1037 (1997).

[25] M. Fuchs, K.S. Schweizer, Macromolecules 30, 5133 (1997); ibid., 5156 (1997).

[26] M. Fuchs, K.S. Schweizer, J. Chem. Phys. 106, 347 (1997).

[27] J.S. Shaffer, J. Chem. Phys. 101, 4205 (1994); ibid 103, 761 (1995).

[28] M.F. Herman, J. Chem. Phys. 92, 2043 (1990); Macromolecules 25, 4925 and 4391 (1992). I. Szleifer and R.F. Loring, J. Chem. Phys. 95, 2080 (1991). J.D. Wilson and R.F. Loring, J. Chem. Phys. 97, 3710 (1992); ibid 99, 7150 (1993).

[29] K.E. Evans, S.F. Edwards, J. Chem. Soc., Faraday Trans. 2, 1891 (1981).

[30] U. Ebert, A. Baumgärtner, L. Schäfer, Phys. Rev. Lett. 78, 1592 (1997).

[31] U. Ebert, L. Schäfer, A. Baumgärtner, J. Stat. Phys. 90, 1325 (1998).

[32] A. Baumgärtner, U. Ebert, L. Schäfer, J. Stat. Phys. 90, 1375 (1998).

[33] L. Schäfer, A. Baumgärtner, U. Ebert, Eur. Phys. J. B 10, 105 (1999).

[34] W. Paul, K. Binder, D.W. Heermann, K. Kremer, J. Phys. II 1, 37 (1991); W. Paul, K. Binder, D.W. Heermann, K. Kremer, J. Chem. Phys. 95, 7726 (1991).

[35] J. Wittmer, W. Paul, K. Binder, Macromolecules 25, 7211 (1992); J. Wittmer, W. Paul, K. Binder, J. Phys. II France 4, 873 (1994). 
[36] M. Müller, J. Wittmer, J.-L. Barrat, On two intrinsic length scales in polymer physics: topological constraints vs. entanglement length, submitted to Europhys. Lett. condmat/0006464].

[37] K. Kremer, K. Binder, Comput. Phys. Reports 7, 259 (1988).

[38] K. Binder, Introduction: General Aspects of Computer Simulation Techniques and Their Applications in Polymer Physics. In: Monte Carlo and Molecular Dynamics Simulations in Polymer Science, edited by K. Binder (Oxford University Press, New York, 1995), pp. 3-46.

[39] H.P. Deutsch, K. Binder, J. Chem. Phys. 94, 2294 (1991).

[40] J. Baschnagel, W. Paul, V. Tries, K. Binder, Macromolecules 31, 3856 (1998).

[41] P.J. Flory, Statistical Mechanics of Chain Molecules (Wiley, New York, 1969).

[42] J.W. Cannon, J.A. Aronovitz, P. Goldbart, J. Phys. I 1, 629 (1991).

[43] J.A. Aronovitz, D.R. Nelson, J. Physique 47, 1445 (1986).

[44] J. Rudnick, G. Gaspari, J. Phys. A: Math. Gen. 19, 191 (1986).

[45] H.W.H.M. Janszen, T.A. Tervoort, P. Cifra, Macromolecules 29, 5678 (1996).

[46] F. Eurich, P. Maass, preprint.

[47] M. Murat, K. Kremer, J. Chem. Phys. 108, 4340 (1998).

[48] J. Baschnagel, K. Binder, P. Doruker, A.A. Gusev, O. Hahn, K. Kremer, W.L. Mattice, F. Müller-Plathe, M. Murat, W. Paul, S. Santos, U.W. Suter, V. Tries, Adv. Polym. Sci. 152, 41 (2000).

[49] P.H. Verdier, J. Chem. Phys 45, 2118 (1966).

[50] G. Allegra, F. Ganazzoli, Adv. Chem. Phys. 75, 265 (1989).

[51] L. Harnau, R.G. Winkler, P. Reineker, Europhys. Lett. 45, 488 (1999).

[52] D. Richter, M. Monkenbusch, J. Allgeier, A. Arbe, J. Colmenero, B. Farago, Y. Cheol Bae, R. Faust, J. Chem. Phys. 111, 6107 (1999).

[53] G.D. Smith, W. Paul, M. Monkenbusch, D. Richter, On the Failure of Some Analytical Models for Chain Motion in Unentangled Polymer Melts, submitted to Phys. Rev. Lett.

[54] R. Faller, Chain stiffness intensifies the reptation characteristics of polymer dynamics in the melt, submitted to Phys. Rev. Lett. cond-mat/0005192.

[55] R. Faller, F. Müller-Plathe, A. Heuer, Local Reorientation Dynamics of Semiflexible Polymers in the Melt, Macromolecules, in press.

[56] K. Kremer, G.S. Grest, J. Chem. Phys. 92, 5057 (1990).

[57] M. Pütz, K. Kremer, G.S. Grest, Europhys. Lett. 49, 735 (2000).

[58] W. Paul, G.D. Smith, Do Y. Yoon, Macromolecules 30, 7772 (1997).

[59] M. Müller, J.P. Wittmer, M.E. Cates, Phys. Rev. E 53, 5063 (1996).

[60] M. Pütz, Dissertation (Mainz, 1999, unpublished).

[61] C. Bennemann, J. Baschnagel, W. Paul, K. Binder, Comp. Theo. Poly. Sci. 9, 217 (1999). 


\section{TABLES}

\begin{tabular}{rrrcrrr}
\hline \hline$N$ & $R_{\mathrm{e}}^{2}$ & $R_{\mathrm{g}}^{2}$ & $\left\langle\lambda_{1}\right\rangle:\left\langle\lambda_{2}\right\rangle:\left\langle\lambda_{3}\right\rangle$ & $\tau_{\mathrm{b}}$ & $\tau_{\mathrm{ee}}$ & $\tau_{1}$ \\
\hline 16 & 136 & 23 & $12.42: 2.78: 1$ & 391 & 15180 & 14519 \\
32 & 293 & 49 & $12.30: 2.74: 1$ & 454 & 78509 & 76879 \\
64 & 607 & 102 & $12.17: 2.71: 1$ & 430 & 367863 & 384993 \\
128 & 1314 & 217 & $11.88: 2.67: 1$ & 445 & 2387464 & 2558552 \\
512 & 5348 & 885 & $11.87: 2.65: 1$ & 461 & 214679565 & 233045839 \\
\hline \hline
\end{tabular}

TABLE I. Chain length dependence of the size of a chain and of its relaxation times: $R_{\mathrm{e}}^{2}=$ mean-square end-to-end vector, $R_{\mathrm{g}}^{2}=$ mean-square radius of gyration, $\left\langle\lambda_{\alpha}\right\rangle=$ mean eigenvalue of the gyration tensor in spatial direction $\alpha(=1,2,3)$ [see Eq. (目)], $\tau_{\mathrm{b}}=$ relaxation time of the bond vector defined by $\phi_{\mathrm{b}}\left(\tau_{\mathrm{b}}\right)=1 / \mathrm{e}, \tau_{\mathrm{ee}}=$ relaxation time of the end-to-end vector defined by Eq. (21), $\tau_{1}=$ relaxation time of the first Rouse mode defined by $\phi_{\mathrm{b}}\left(\tau_{\mathrm{b}}\right)=1 / \mathrm{e}$. 


\section{FIGURES}

FIG. 1. Mean-square end-to-end vector, $R_{\mathrm{e}}^{2}$ (upper symbols), and radius of gyration, $R_{\mathrm{g}}^{2}$ (lower symbols), plotted against chain length $N$. Asymptotically, both quantities are expected to behave as $R_{\mathrm{e}}^{2}=C_{\infty} l^{2}(N-1)$ and $R_{\mathrm{g}}^{2}=C_{\infty} l^{2}(N-1) / 6$ for dense melts. Here, $l=\left\langle\boldsymbol{l}^{2}\right\rangle^{1 / 2}(=2.64)$ is the mean bond length and $C_{\infty}$ the characteristic ratio at infinite chain length. The value of $C_{\infty} \simeq 1.56$ was obtained by an extrapolation of $R_{\mathrm{e}}^{2} /(N-1) l^{2}$ to $N \rightarrow \infty$ using $N>100$. Both $R_{\mathrm{e}}^{2}$ and $R_{\mathrm{g}}^{2}$ enter the asymptotic regime if $N>100$ (for $R_{\mathrm{g}}^{2}$ this is hard to see on the scale of this figure; see Fig. 2). Furthermore, the figure shows that the present data are consistent with previously obtained results (on smaller systems) 34, 35.

FIG. 2. Eigenvalues, $\left\langle\lambda_{\alpha}\right\rangle /(N-1)(\alpha=1,2,3)$, of the gyration tensor Q [Eq. (11)] plotted versus chain length $N$. If the shape of a polymer were spherical, one would have $\left\langle\lambda_{1}\right\rangle=\left\langle\lambda_{2}\right\rangle=\left\langle\lambda_{3}\right\rangle$. The result $\left\langle\lambda_{1}\right\rangle \gg\left\langle\lambda_{2}\right\rangle>\left\langle\lambda_{3}\right\rangle$ shows that it rather resembles a flattened ellipsoid ("prolate" chains). The ratio of the eigenvalues $\left(\left\langle\lambda_{1}\right\rangle:\left\langle\lambda_{2}\right\rangle:\left\langle\lambda_{3}\right\rangle=11.87: 2.65: 1\right.$ for $\left.N=512\right)$ is fairly close to that expected for random walks (12.03:2.72:1 [45]). As anticipated from Eq. (2), the eigenvalues sum up to the radius of gyration calculated independently by $R_{\mathrm{g}}^{2}=\sum_{n=1}^{N}\left(\boldsymbol{R}_{n}-\boldsymbol{R}_{\mathrm{cm}}\right)^{2} / N$, where $\boldsymbol{R}_{n}$ and $\boldsymbol{R}_{\mathrm{cm}}$ are the position vectors of the $n$th monomer and of the center of mass, respectively.

FIG. 3. Rescaled static autocorrelation of the Rouse modes, $8 N(N-1)\left\langle\boldsymbol{X}_{p}^{2}(0)\right\rangle / R_{\mathrm{e}}^{2}$, versus $p / N$ for all chain lengths studied $\left(R_{\mathrm{e}}=\right.$ end-to-end distance). This rescaling is motivated by Eq. (4). The dashed and the solid lines shows the theoretical predictions for fully flexible chains [Eq. (1)] and for freely rotating chains [Eq. (5)] where local stiffness due to the bond angle is taken into account. If $p / N \lesssim 0.05$ (corresponding to subchains larger than about 20 monomers), both predictions coincide and describe the simulation data well. In this case, Eqs. (田) and (5) are well approximated by the power law $p^{-2}$ (indicated by a solid line in the figure). However, if $p / N>0.3$ (corresponding to subchains smaller than about 3 monomers), the fully flexible (standard) Rouse model overestimates the correlation, whereas the (approximate) consideration of chain stiffness still provides a reasonable description. A magnification of the comparison between Eqs. (4) and (5) is shown in the inset for $N=512$.

FIG. 4. Test of the orthogonality of the Rouse modes at $t=0$ [see Eq. (㺼)]. Figure (a) shows $\left\langle\boldsymbol{X}_{1}(0) \cdot \boldsymbol{X}_{q}(0)\right\rangle(q=1, \ldots, 127)$ for $N=128\left(>N_{\mathrm{e}} \approx 37 ; N_{\mathrm{e}}=\right.$ entanglement length). The inset magnifies the results for $q=2, \ldots, 127$. Cross-correlations between the first and the $q=2,3$ modes are about 1 to 2 orders of magnitude smaller than $\left\langle\boldsymbol{X}_{1}^{2}(0)\right\rangle$, but much stronger than those for $q>3$. If $N<N_{\mathrm{e}}$, the behavior is qualitatively the same as shown in the inset. However, all correlations are more than 2 orders of magnitude smaller than the self-correlation for non-entangled chains. Figure (b) compares $\left\langle\boldsymbol{X}_{p}(0) \cdot \boldsymbol{X}_{q}(0)\right\rangle$ of the largest mode for $N=128,\left\langle\boldsymbol{X}_{127}(0) \cdot \boldsymbol{X}_{q}(0)\right\rangle$, with that for $N=16\left(<N_{\mathrm{e}}\right),\left\langle\boldsymbol{X}_{15}(0) \cdot \boldsymbol{X}_{q}(0)\right\rangle$. If $N>N_{\mathrm{e}}$, cross-correlations with the smallest Rouse modes are as large as the self-correlation, whereas they are at least an order of magnitude smaller if $N<N_{\mathrm{e}}$. 
FIG. 5. Time dependence of $g_{6}(t) / t^{1 / 4}$ for different chain lengths $N . g_{6}(t)$ is the cubic invariant defined by Eq. (9). This function is predicted to behave similarly to the mean-square displacement of an inner monomer $g_{1}(t)$, but to exhibit the characteristics of reptation more clearly than $g_{1}(t)$ [30 33]. In the interval $200 \lesssim t \lesssim 5000, g_{6}(t)$ increases roughly as $t^{1 / 2}$. Then, it crosses over to a weaker time-dependence which is compatible with $g_{6}(t) \sim t^{1 / 4}$ for $4 \times 10^{5} \lesssim t \lesssim 2 \times 10^{6}$. The intersection between these two asymptotic power-laws provides an estimate for the entanglement time $\tau_{\mathrm{e}} \approx 100175$ (solid vertical line). Using Eq. (10), $g_{6}\left(\tau_{\mathrm{e}}\right) / \tau_{\mathrm{e}}^{1 / 4} \simeq d_{\mathrm{T}}^{2} / 3 \tau_{\mathrm{e}}^{1 / 4} \approx 7$, the tube diameter is approximately given by $d_{\mathrm{T}} \approx 19.3$. Furthermore, the two dashed vertical lines indicate the values of the relaxation time, $\tau_{\text {ee }}$, of the end-to-end vector, defined by Eq. (21), for $N=16$ and $N=512$.

FIG. 6. Time dependence of $g_{2}(t) / t^{1 / 4}$ for different chain lengths $N . g_{2}(t)$ is the displacement of an inner monomer relative to the chain's center of mass [Eq. (6)]. For times shorter than the Rouse, $\tau_{\mathrm{R}}$, or disentanglement time, $\tau_{\mathrm{d}}$, one expects $g_{2}(t) \sim g_{1}(t)$, whereas $g_{2}(t) \rightarrow R_{\mathrm{g}}^{2}$ otherwise. Due to Eq. (22) these times can be represented by the relaxation time, $\tau_{\text {ee }}$, of the end-to-end vector [defined by Eq. (21)]. The latter behavior is borne out for $t>\tau_{\text {ee }}$ (vertical dashed lines shown for $N=16$ and 512 only). As in Fig. 5 for $g_{6}\left(\approx g_{1}\right)$, there is an approximate $t^{1 / 2}$-increase for $200 \lesssim t \lesssim 5000$ which crosses over to a weaker time dependence. This weaker dependence is (perhaps) compatible with $g_{2} \sim t^{1 / 4}$ for $N=512$ over about half a decade $\left(5 \times 10^{5} \lesssim t \lesssim 10^{6}\right)$. Using this interval the intersection with the extrapolation of the $t^{1 / 2}$-behavior yields estimates for the entanglement time, $\tau_{\mathrm{e}}$, and the tube diameter, $d_{\mathrm{T}}: \tau_{\mathrm{e}} \approx 84053$ (solid vertical line), $d_{\mathrm{T}} \approx 18.6$ [via Eq. (10)].

FIG. 7. Ratio of the mean-square displacement of the end monomer, $g_{4}(t)$, and the inner monomer, $g_{1}(t)$, versus logarithm of time for different chain lengths $N$ [see Eq. (6))]. The dashed vertical lines indicate the relaxation time, $\tau_{\text {ee }}$, of the end-to-end vector for $N=16$ and $N=128\left[\tau_{\text {ee }}\right.$ is defined by Eq. (21); see Table []. The vertical dotted shows the value of the maximum, $g_{4} / g_{1}=2$, expected from the Rouse model. This maximum should occur for $t \lesssim \tau_{\mathrm{R}}$. For a reptating chain, a maximum is still expected to occur at $t \approx \tau_{\mathrm{R}}$, but to be much larger than 2 [31]33]. The onset of this behavior is visible for $N=128$. In the limit $t \rightarrow \infty$, both displacements become diffusive. So, $g_{4} / g_{1} \rightarrow 1$. Since $g_{4} \sim g_{1}$ in general, the calculation of the ratio $g_{4} / g_{1}$ eliminates the dominant variation with time and highlights the differences between both displacements. Thereby, statistical errors also become much better visible. For $N=512$, they are so pronounced that a quantitative analysis was not possible. 
FIG. 8. Time dependence of $g_{3}(t) / t^{3 / 4}$ for different chain lengths $N . g_{3}(t)$ is the mean-square displacement of the chain's center of mass [Eq. (6)]. Since reptation theory predicts a crossover from $g_{3} \sim t^{1 / 2}$ for $\tau_{\mathrm{e}} \lesssim t \lesssim \tau_{\mathrm{R}}$ to $g_{3} \sim t$ for $t>\tau_{\mathrm{R}}, g_{3}(t) / t^{3 / 4}$ should exhibit a minimum at $t \simeq \tau_{\mathrm{R}}$ and increase as $t^{-1 / 4}$ and $t^{1 / 4}$ for $t<\tau_{\mathrm{R}}$ and $t>\tau_{\mathrm{R}}$, respectively. Qualitatively, such a behavior is observed for $N=512$ only (solid lines with slope $1 / 2$ and 1 ). The intersection point of both power laws yields an estimate for the Rouse time $\tau_{\mathrm{R}} \approx 4.34 \times 10^{6}$, whence $d_{\mathrm{T}} \approx 13$ by virtue of $g_{3}\left(\tau_{\mathrm{R}}\right)=d_{\mathrm{T}}^{2} / 3$. The minimum is preceeded by another power-law increase (empirically, $g_{3} \sim t^{0.84}$ ) if $t \lesssim \tau_{\mathrm{e}}$. For smaller chain lengths the power law is not as well pronounced. The intersection point of the power laws $g_{3} \sim t^{0.84}$ and $g_{3} \sim t^{1 / 2}$ yields another estimate of the entanglement time, $\tau_{\mathrm{e}} \approx 268718$, and of the tube diamater, $d_{\mathrm{T}} \approx 20.3$ [see Eq. (13)]. $g_{3}$ becomes diffusive in the range $t \approx \tau_{\text {ee }}$ [indicated by dashed vertical lines for $N=16$ and $512 ; \tau_{\text {ee }}=$ relaxation time of the end-to-end vector, see Eq. (21)].

FIG. 9. Scaling plot of the autocorrelation function, $\phi_{\mathrm{b}}(t)$, of the bond vectors for different chain lengths versus $t / \tau_{\mathrm{b}}$. The relaxation time, $\tau_{\mathrm{b}}$, was defined by (see Table $\mathbb{\mathbb { Z }}$ ): $\phi_{\mathrm{b}}\left(\tau_{\mathrm{b}}\right)=1 / \mathrm{e}$ (dashed horizontal lines in the main figure and the inset). This scaling collapses the correlators onto a master curve if $0.25 \lesssim \phi_{\mathrm{b}}(t) \leq 1$. The final decay, $\phi_{\mathrm{b}}(t)<0.25$, depends on chain length. For $N=16$, it is well described by the Rouse model, i.e., by Eq. (15) (dashed curve in the main figure), whereas Eq. (15) decays faster than the simulation data for $N \geq 32$. Especially, the largest chain lengths $(N=128,512)$ exhibit a slow power-law decay (emprically, $\left.\phi_{\mathrm{b}}(t) \sim t^{-0.35}\right)$, as illustrated in the inset. The inset also indicates the Rouse behavior, $\phi_{\mathrm{b}}(t) \sim t^{-0.5}$, expected for $t<\tau_{\mathrm{e}}\left(\tau_{\mathrm{e}}=92114=\right.$ average entanglement time of Figs. 司 and (6) $)$.

FIG. 10. Survival probability (17), $\phi_{n}(t)$, of an initial bond vector $\boldsymbol{l}_{n}(0)$ for $N=16$ [panel (a)] and $N=128$ [panel (b)]. $\phi_{n}(t)$ is plotted as a function of the bond number $n$. For example, $n=1$ corresponds to the bond vector $\boldsymbol{l}_{1}(0)$ connecting the first and the second monomer. Time is measured in units of the relaxation time of the end-to-end vector $\tau_{\text {ee }}$ (see Table (I)). The solid lines represent the prediction of Eq. (18). To improve the statistics the simulation results were averaged over both halves of the chain - the chain is symmetric with respect to its ends. Therefore, results from $n=1$ to $n=N / 2$ are shown only. This was sufficient for the short chain, but not for $N=128$. In this case, the bonds $n$ and $n+1$ were lumped additionally and the resulting average was plotted at the (non-existing) bond number $n+1 / 2$.

FIG. 11. Autocorrelation function of the end-end vector, $\phi_{\mathrm{e}}(t)$, versus scaled time $t / \tau_{\mathrm{ee}}$ for all chain lengths studied. The scaling time, $\tau_{\mathrm{ee}}$, is defined by Eq. (21): $\phi_{\mathrm{e}}\left(\tau_{\mathrm{ee}}\right)=0.298221$ (dashed horizontal line). The vertical dashed line indicate the value of the entanglement time $\tau_{\mathrm{e}}(=92114=$ average $\tau_{\text {ee }}$ from Figs. 5 and 6) and of the Rouse time $\tau_{\mathrm{R}}(N=512)\left[\approx 4.34 \times 10^{6}\right]$ determined from Fig. 8. Both times are divided by $\tau_{\text {ee }}(N=512)$ (see Table $\mathbb{E}$ ). The solid line depicts the result of Eq. (20). The inset shows $1-\phi_{\mathrm{e}}(t)$ versus $t / \tau_{\text {ee }}$ for $N=512$ together with the power laws $1-\phi_{\mathrm{e}}(t) \sim t^{9 / 32}$ and $1-\phi_{\mathrm{e}}(t) \sim t^{3 / 8}$ expected from polymer mode-coupling theory for $\tau_{\mathrm{e}} \ll t \ll \tau_{\mathrm{R}}$ and $\tau_{\mathrm{R}} \ll t \ll \tau_{\mathrm{RR}}\left(\tau_{\mathrm{RR}}=\right.$ renormalized Rouse time) [24]. 
FIG. 12. Correlation function of the Rouse modes, $\phi_{p}(t)$, versus rescaled time $t / \tau_{p}$ for $N=16$ [panel (a)] et $N=512$ [panel (b)]. The scaling time, $\tau_{p}$, is defined by: $\phi_{p}\left(\tau_{p}\right)=1 / \mathrm{e}$ (dashed horizontal lines). For both chain lengths the relaxation of four representative mode indices $p$ $(=1, \ldots, N-1)$ is shown. In addition, an exponential function (solid lines) is depicted. This is the behavior expected from the Rouse model [see Eq. (16)]. The vertical dashed line in panel (b) indicates the Rouse time $\tau_{\mathrm{R}}(N=512)\left[\approx 4.34 \times 10^{6}\right]$ determined from Fig. 8 . $\tau_{\mathrm{R}}$ is divided by the relaxation time, $\tau_{1}$, of the first Rouse mode (see Table đI).

FIG. 13. Variation of the Rouse mode relaxation time with the mode index $p$ for different chain lengths $N$ (entanglement length $N_{\mathrm{e}} \approx 37$ ). The relaxation time, $\tau_{p}$, is defined by the time value at which the correlation function of the Rouse modes has decayed to $1 /$ e, i.e., $\phi_{p}\left(\tau_{p}\right)=1$ /e. Motivated by Rouse theory the abscissa and ordinate are scaled according to Eq. (23) where $\zeta b^{2} / k_{\mathrm{B}} T$ is identified with $R_{\mathrm{e}}^{2} / N(N-1) D$ ( $D=$ diffusion coefficient, see Eq. (25) $; R_{\mathrm{e}}^{2}=$ mean-square end-to-end vector). This scaling leads to a reasonable collapse if $N \leq 64$, whereas the $p$-dependence for $N=128,512\left(\gg N_{\mathrm{e}}\right)$ is s-shaped. $\tau_{p}$ is larger than the Rouse prediction (solid line) if $p / N$ is small (especially for $N=512$ ), but smaller than it for $p / N \rightarrow 1$. As for the static correlation $\left\langle\boldsymbol{X}^{2}(0)\right\rangle$ (see Fig. 3), the Rouse model provides a good description of the $p$-dependence if $p / N \lesssim 0.05$ (and $N \leq 64)$, but overestimates the relaxation time for $p / N>0.3$. The inset therefore tests whether $\tau_{p}$ can be written as $\tau_{p}=2\left\langle\boldsymbol{X}^{2}(0)\right\rangle / 3 D$ [see Eq. (24)] with $\left\langle\boldsymbol{X}^{2}(0)\right\rangle$ taken from the simulation (see Fig. 3). This is possible for $N \leq 64$, but not for large (entangled) chains, such as $N=512$.

FIG. 14. Chain length dependence of the end-to-end vector relaxation time, $\tau_{\text {ee }}$ (main figure), and of the diffusion coefficient, $D$, of a chain (inset). $\tau_{\text {ee }}$ and $D$ are determined from Eq. (21) and from the long-time limit of $g_{3}\left(=\lim _{t \rightarrow \infty} g_{3}(t) / 6 t\right)$, respectively. Both quantities are scaled by the $N$-dependences of Eqs. (22) and (25) to highlight deviations from Rouse behavior. The diffusion coefficient is compared with results from previous studies [34,35]. The solid lines in the main figure and the inset indicate the behavior, $\tau_{\text {ee }} \sim N^{3}$ and $D \sim N^{-2}$, suggested by reptation theory. The triangles $(\triangle)$ are the predictions of the Rouse formula $\tau_{\mathrm{ee}}=(N b)^{2} \zeta / 3 \pi^{2} k_{\mathrm{B}} T=R_{\mathrm{e}}^{2} / 3 \pi^{2} D$, where the simulation results are used for $D$ and $R_{\mathrm{e}}^{2}$ (= end-to-end distance). 


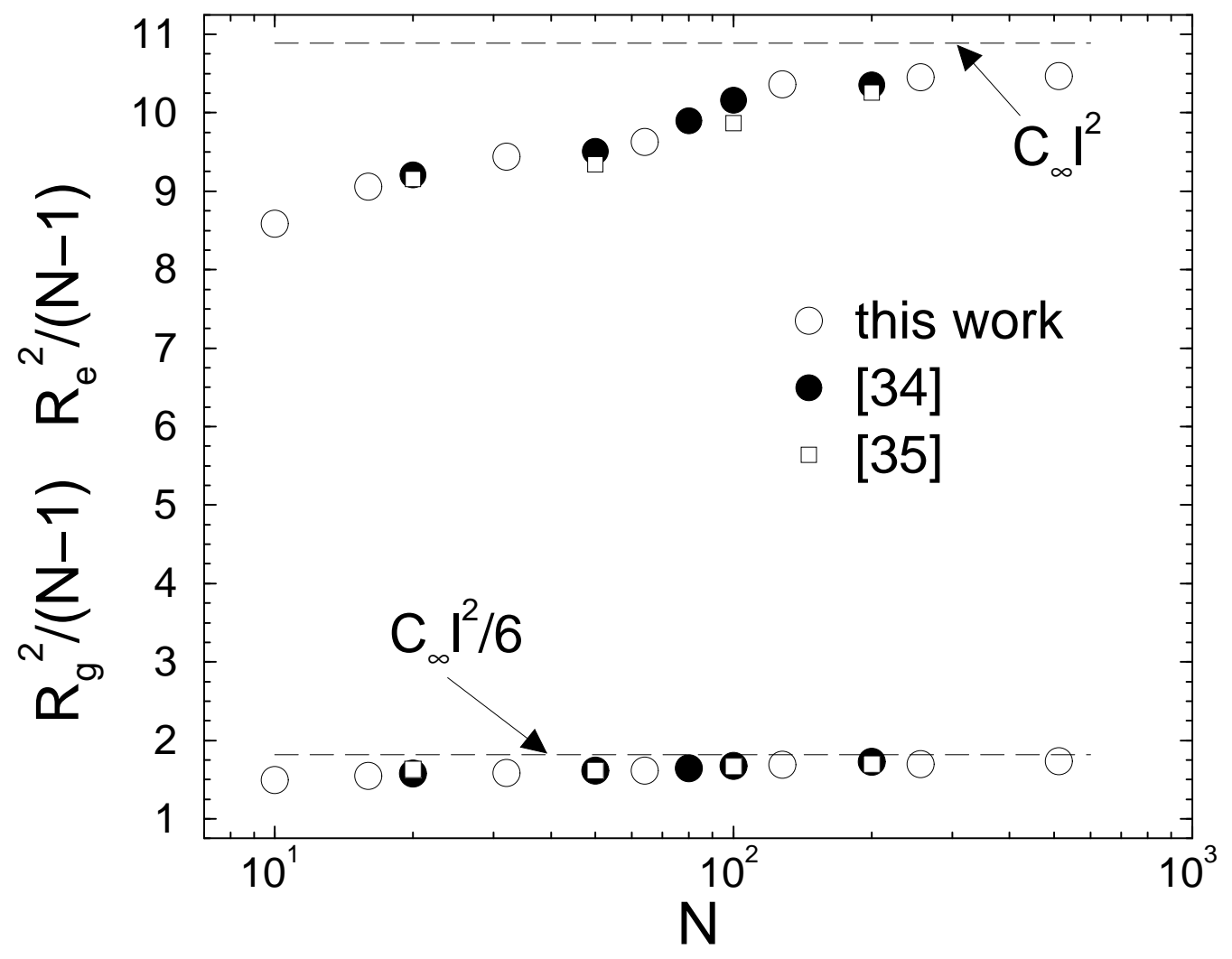

FIG. 1. 


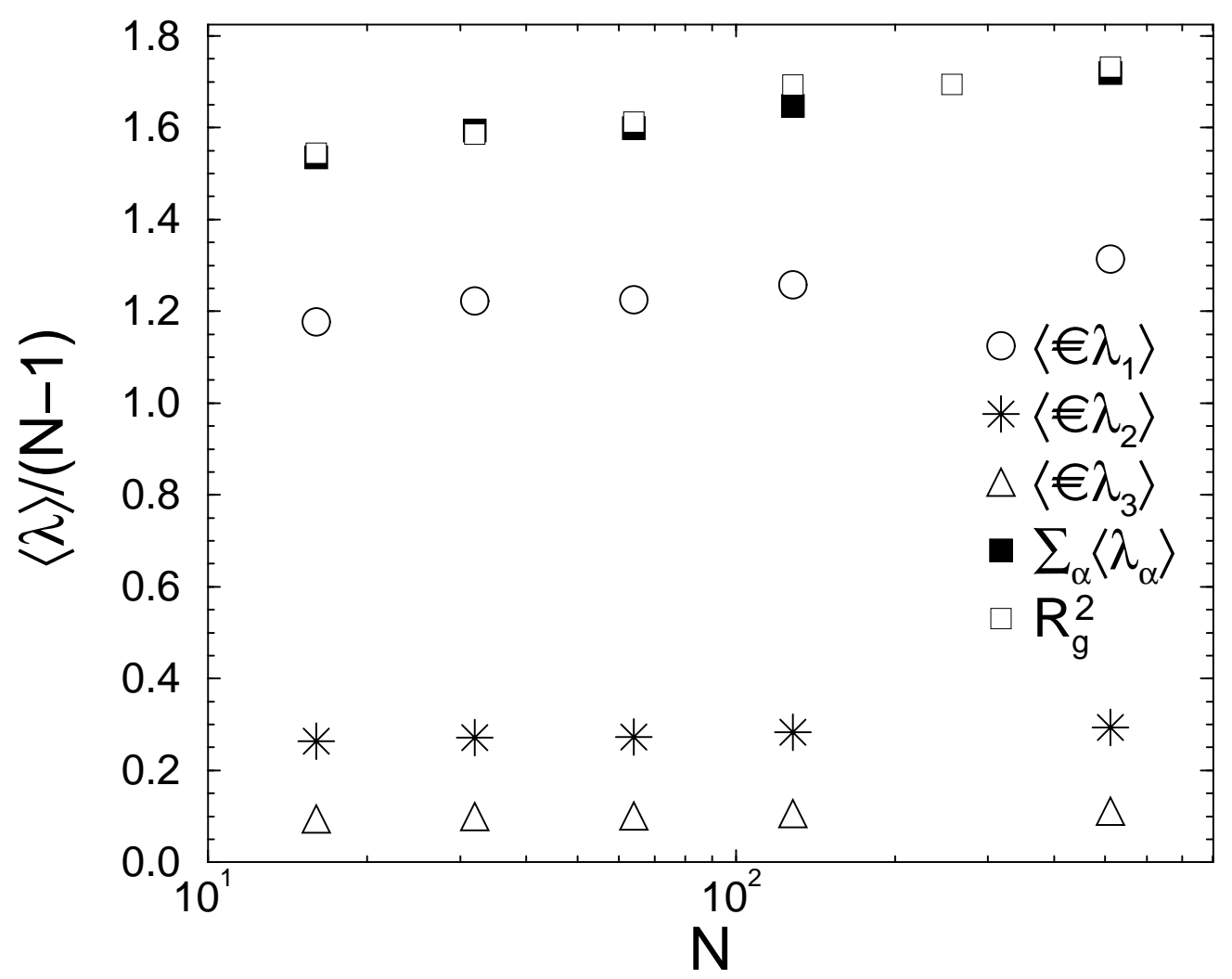

FIG. 2. 


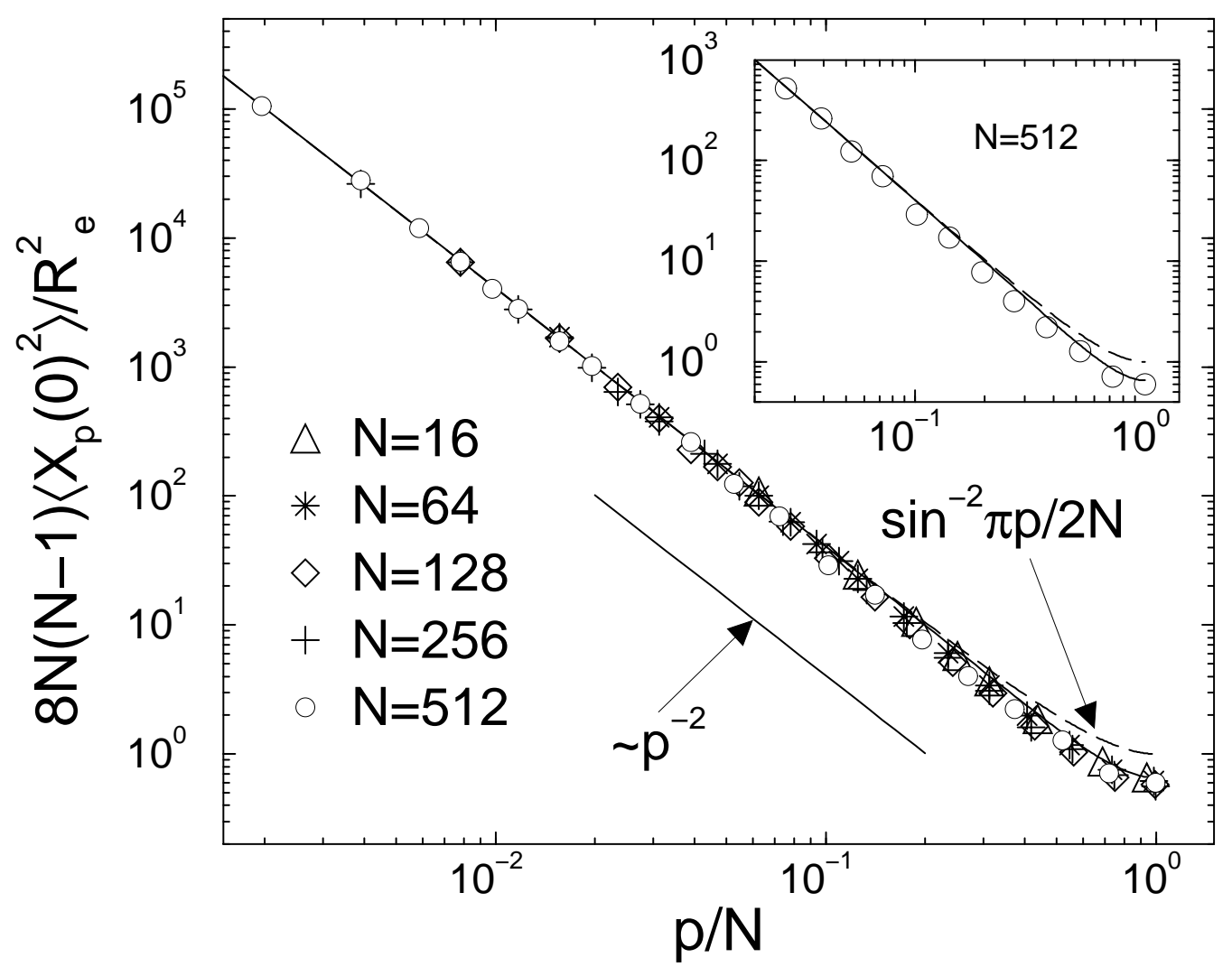

FIG. 3. 


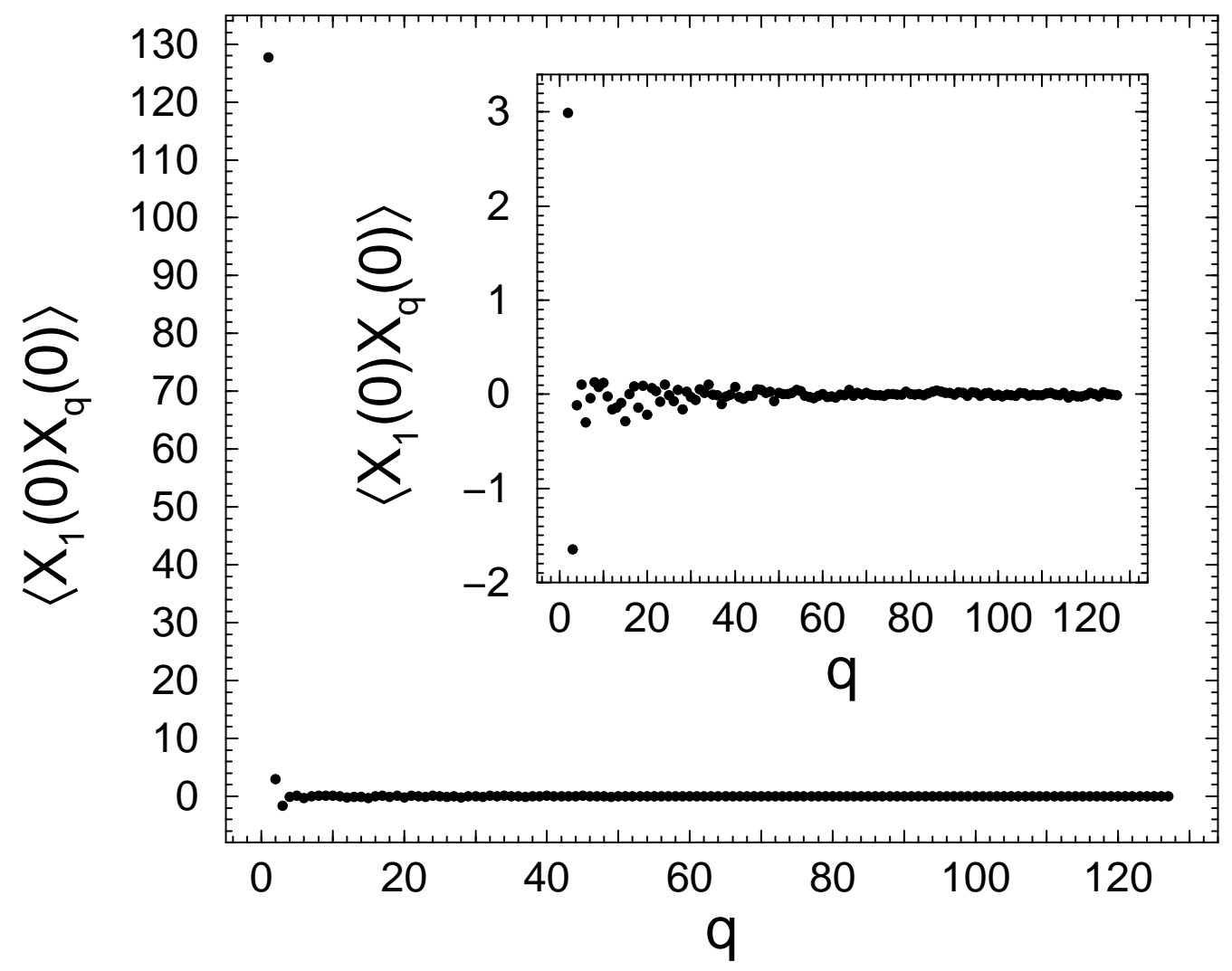

(a)

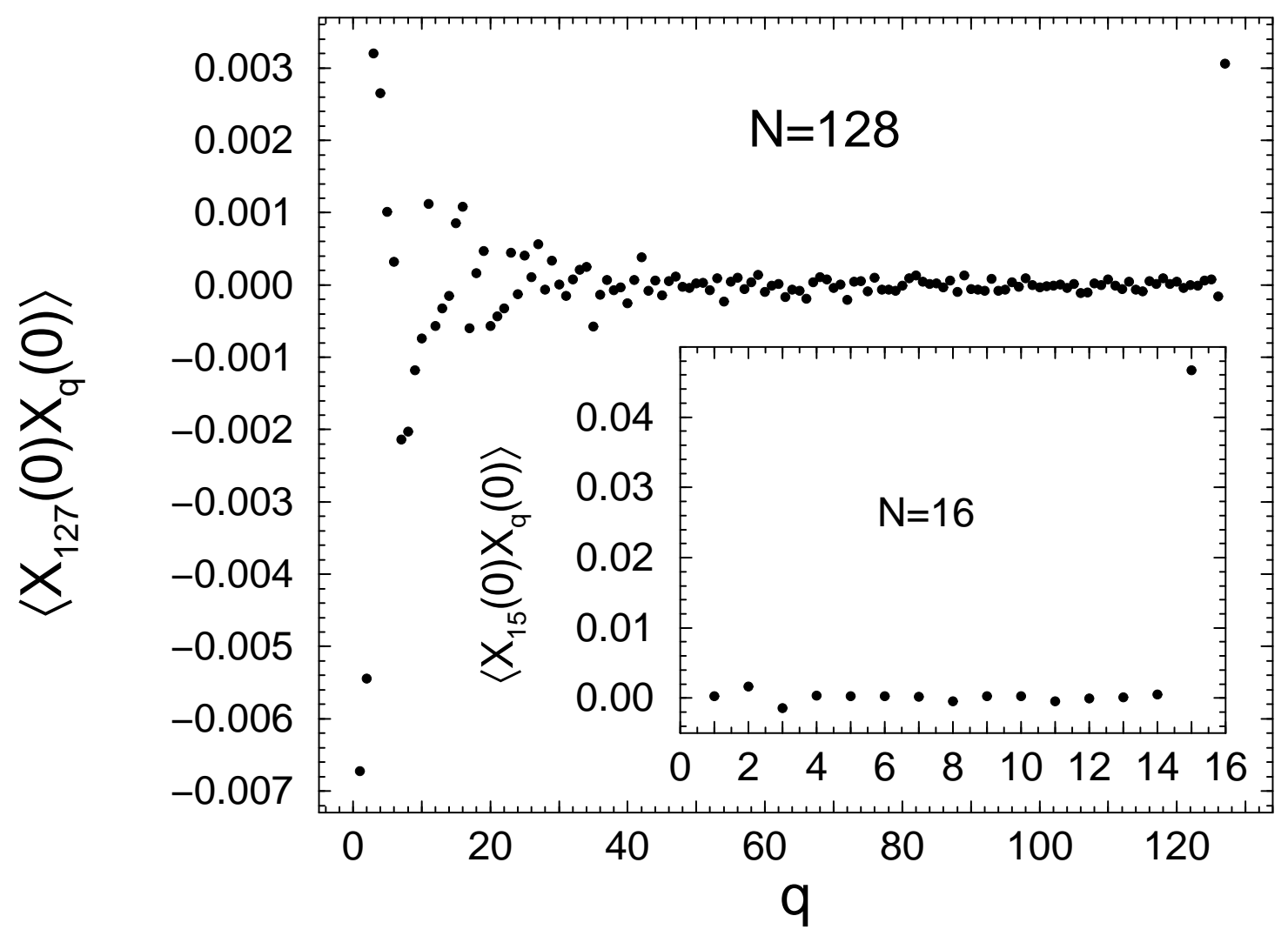

(b)

FIG. 4. 


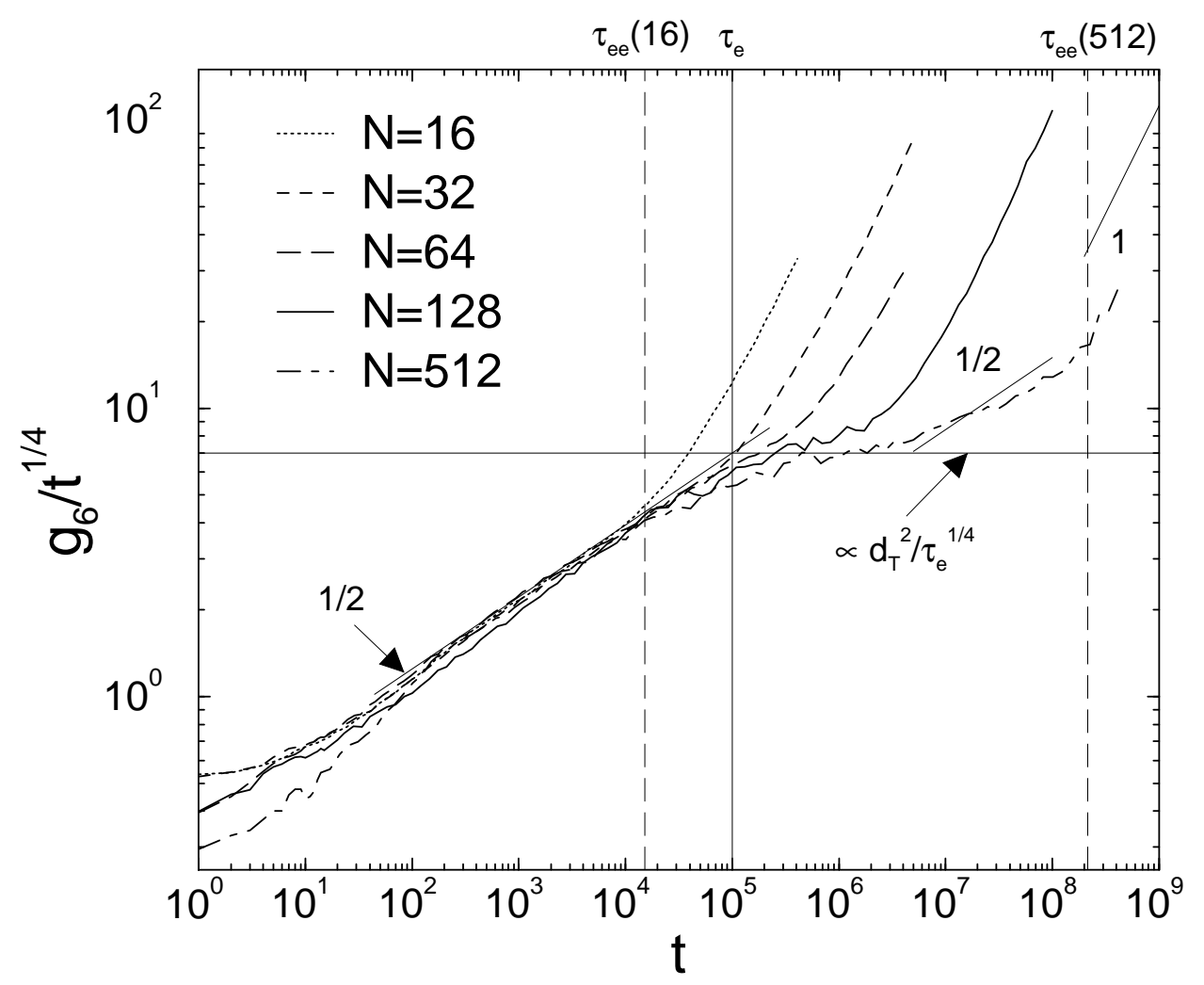

FIG. 5. 


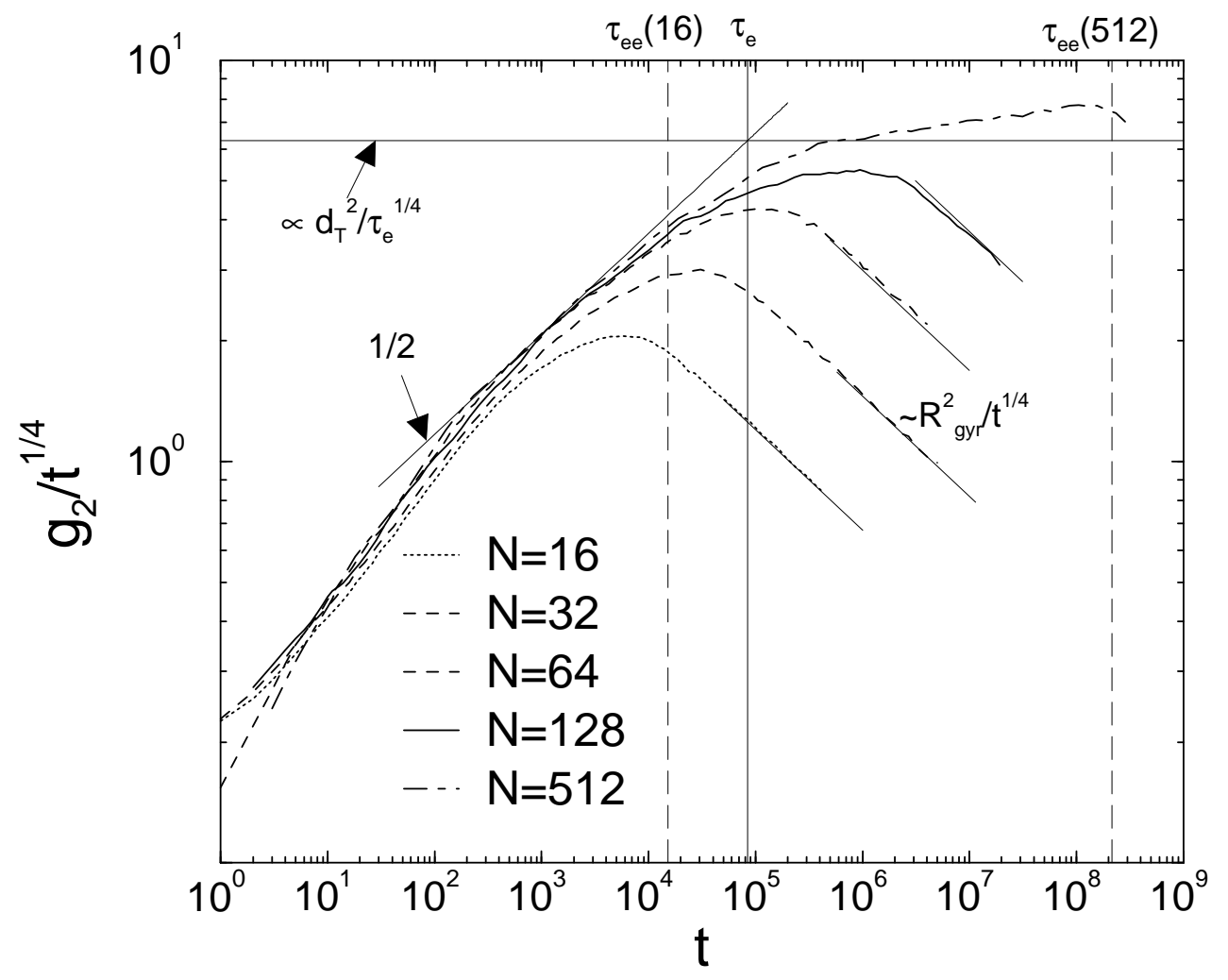

FIG. 6. 


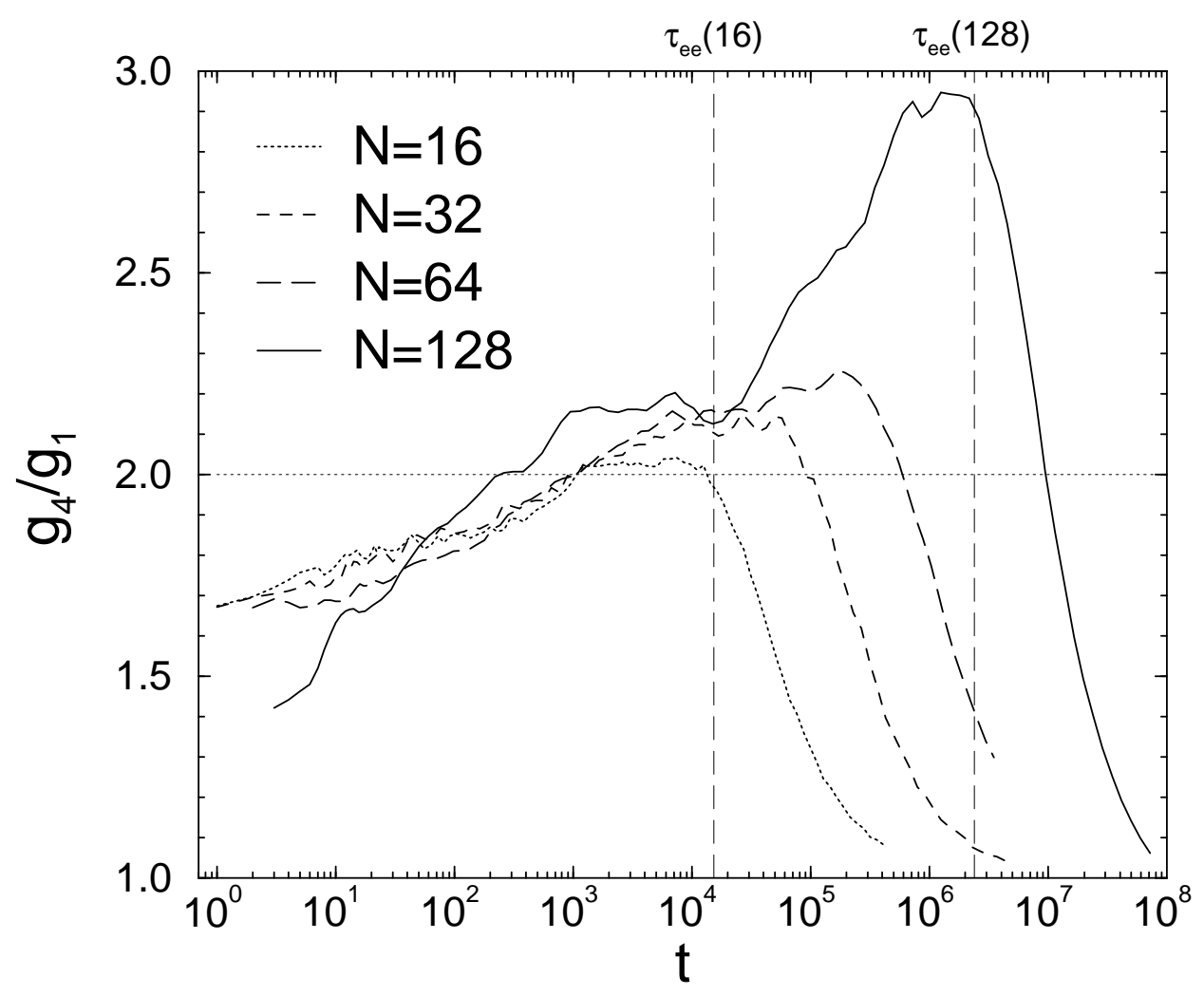

FIG. 7. 


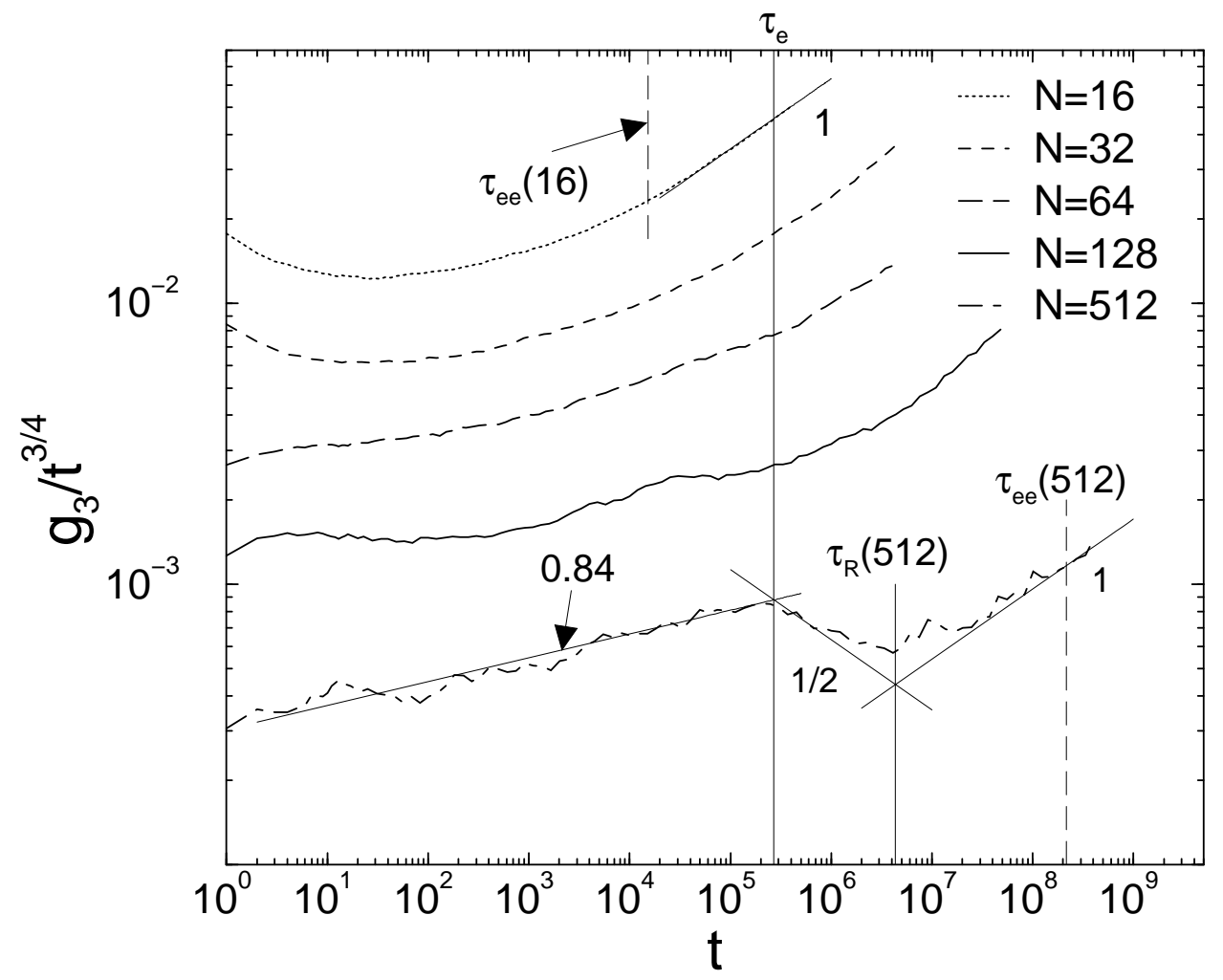

FIG. 8. 


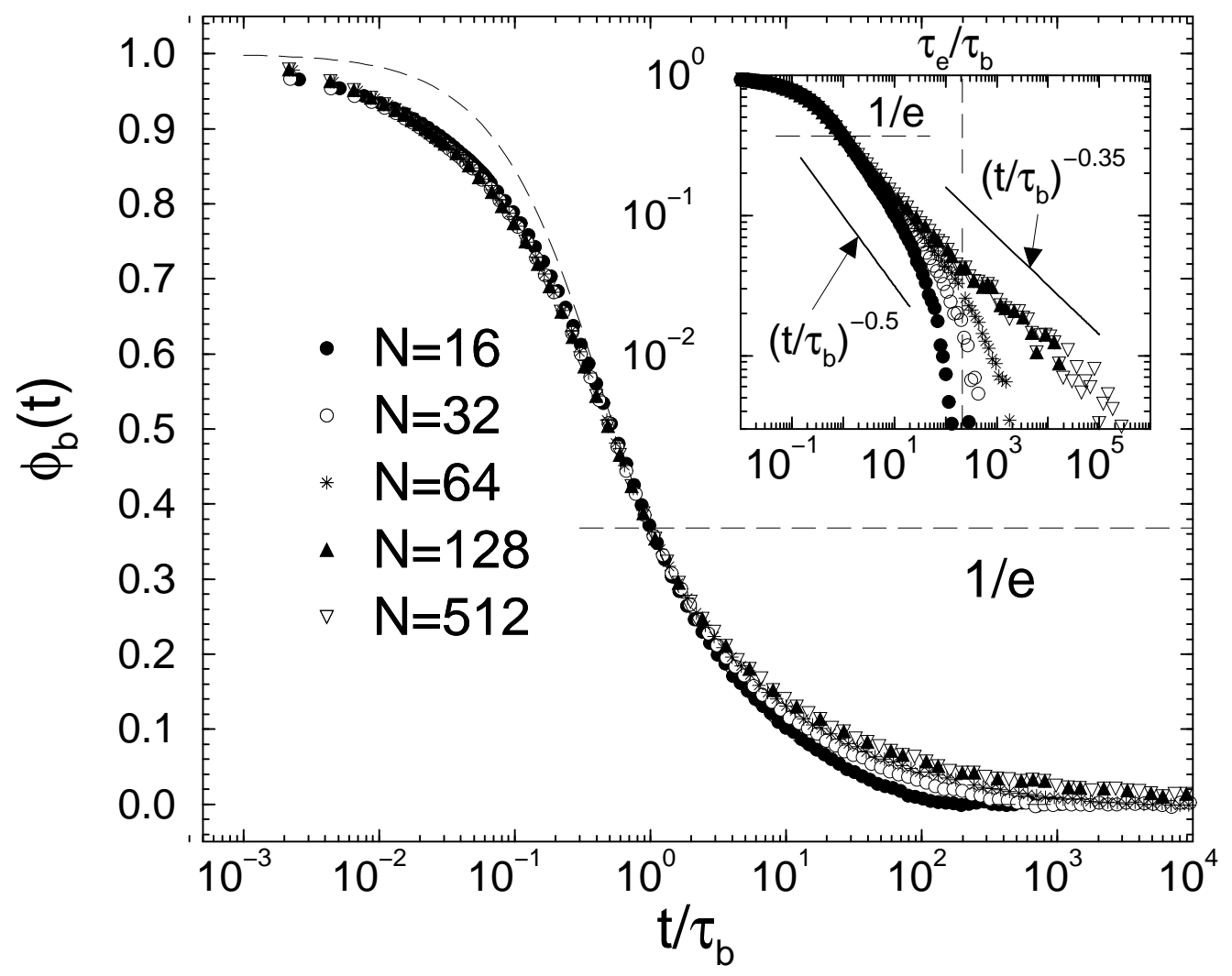

FIG. 9. 


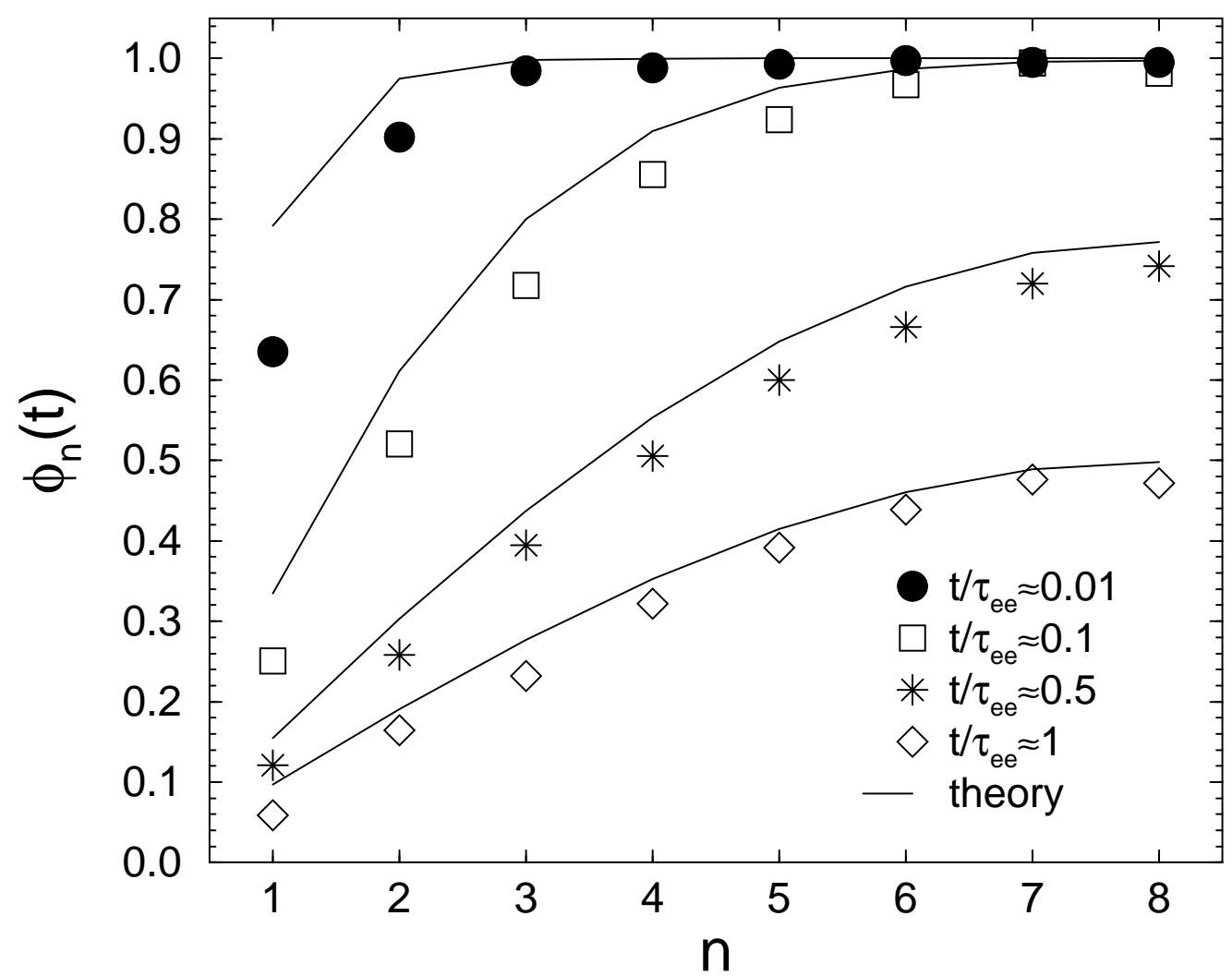

(a)

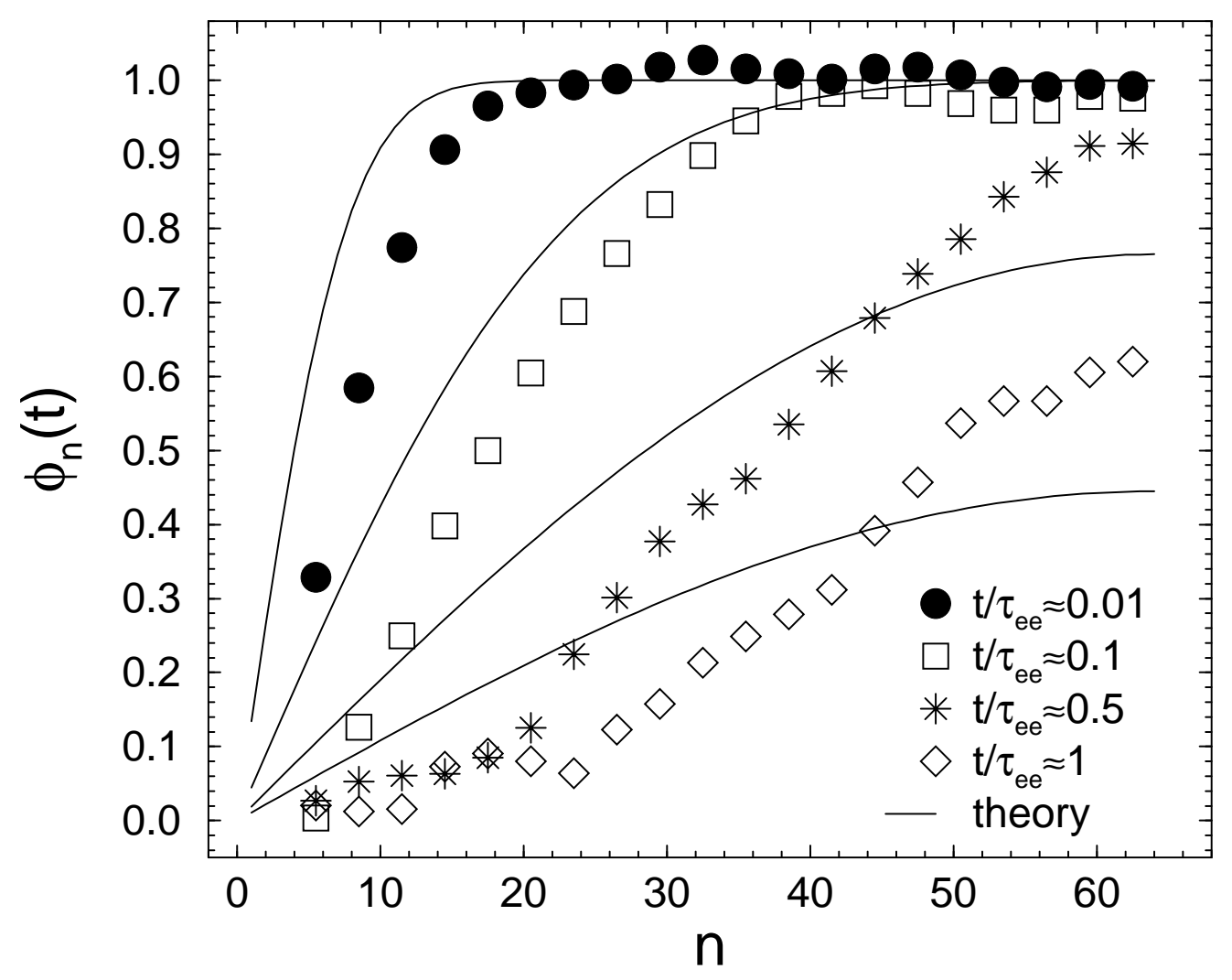

(b)

FIG. 10. 


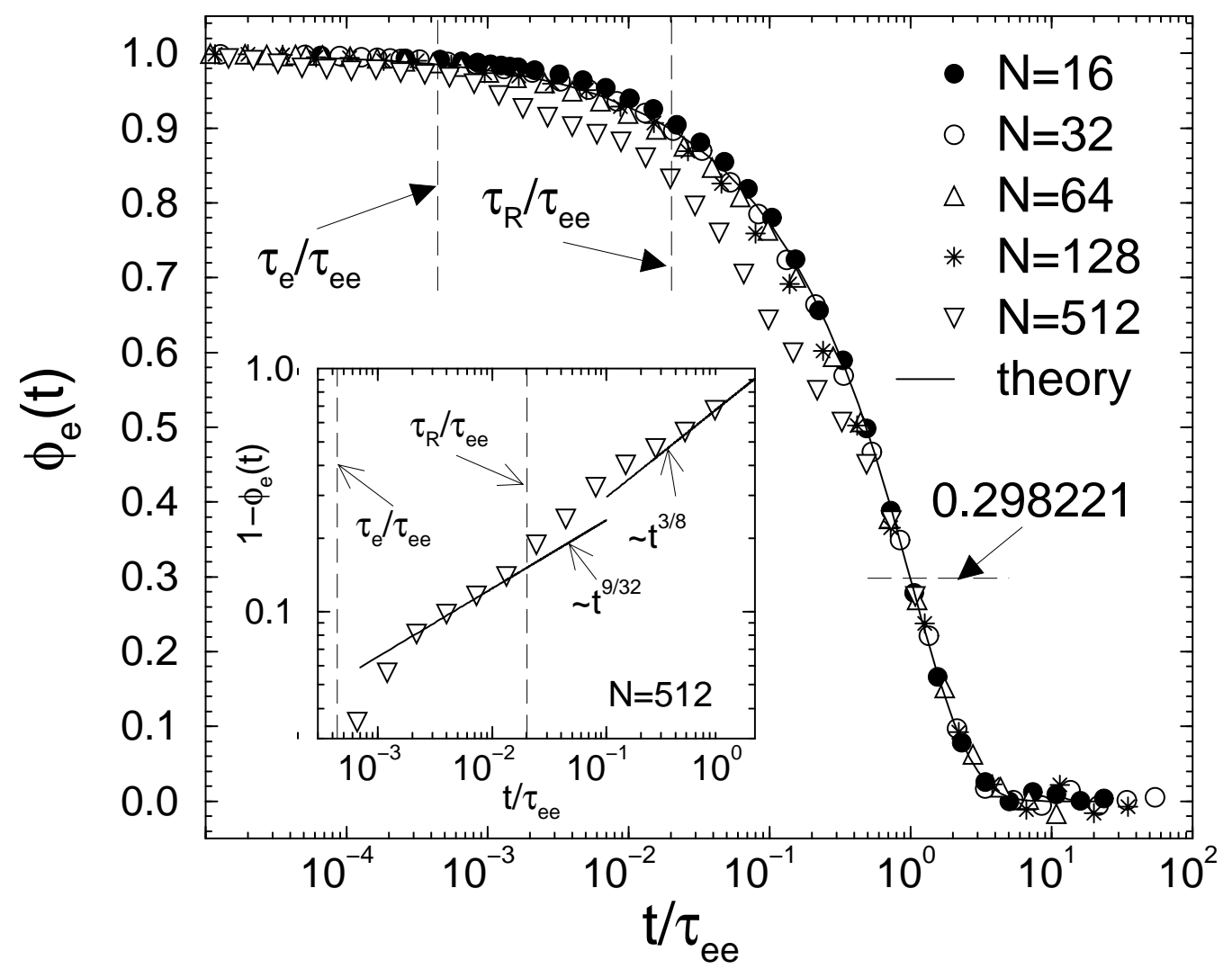

FIG. 11. 


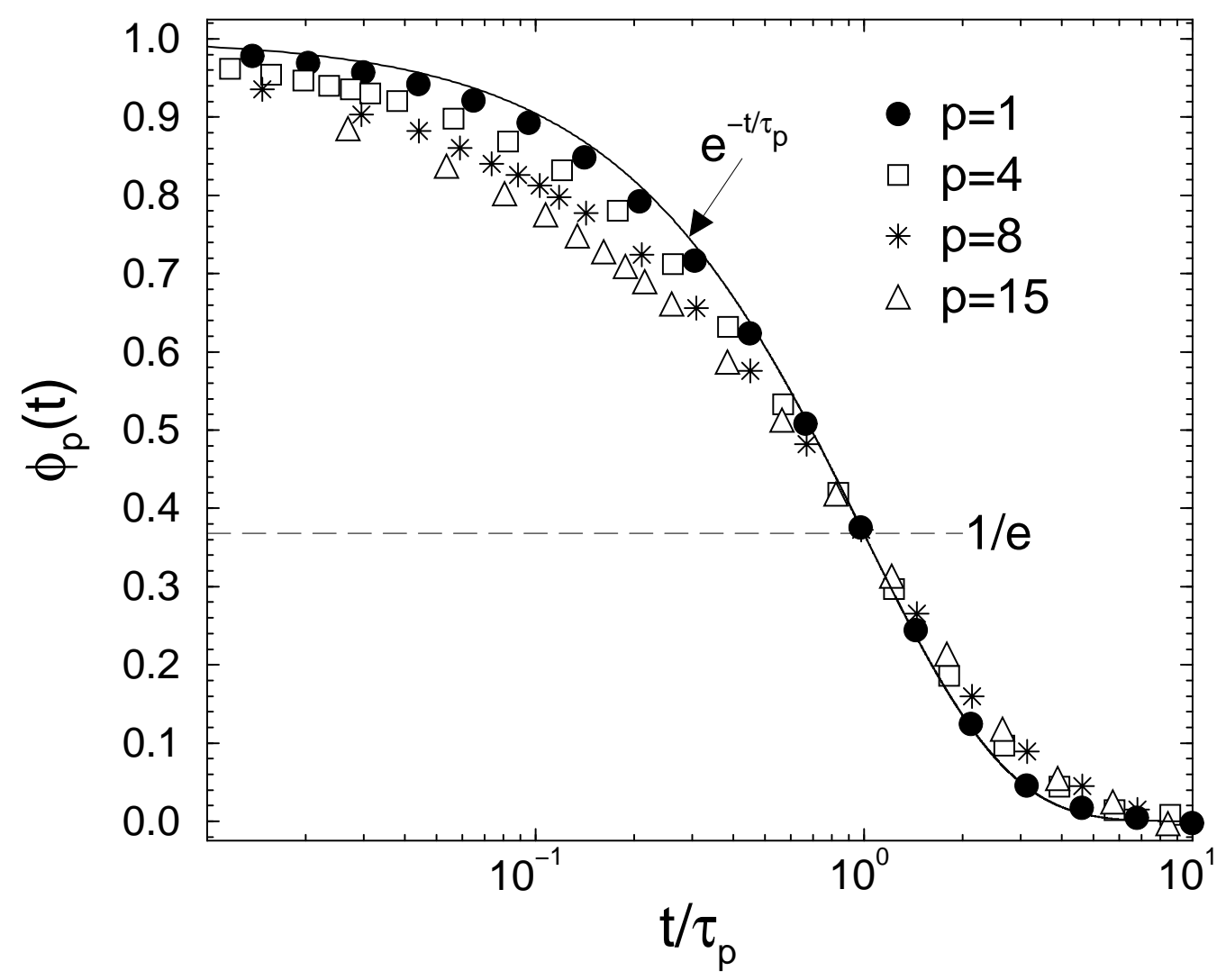

(a)

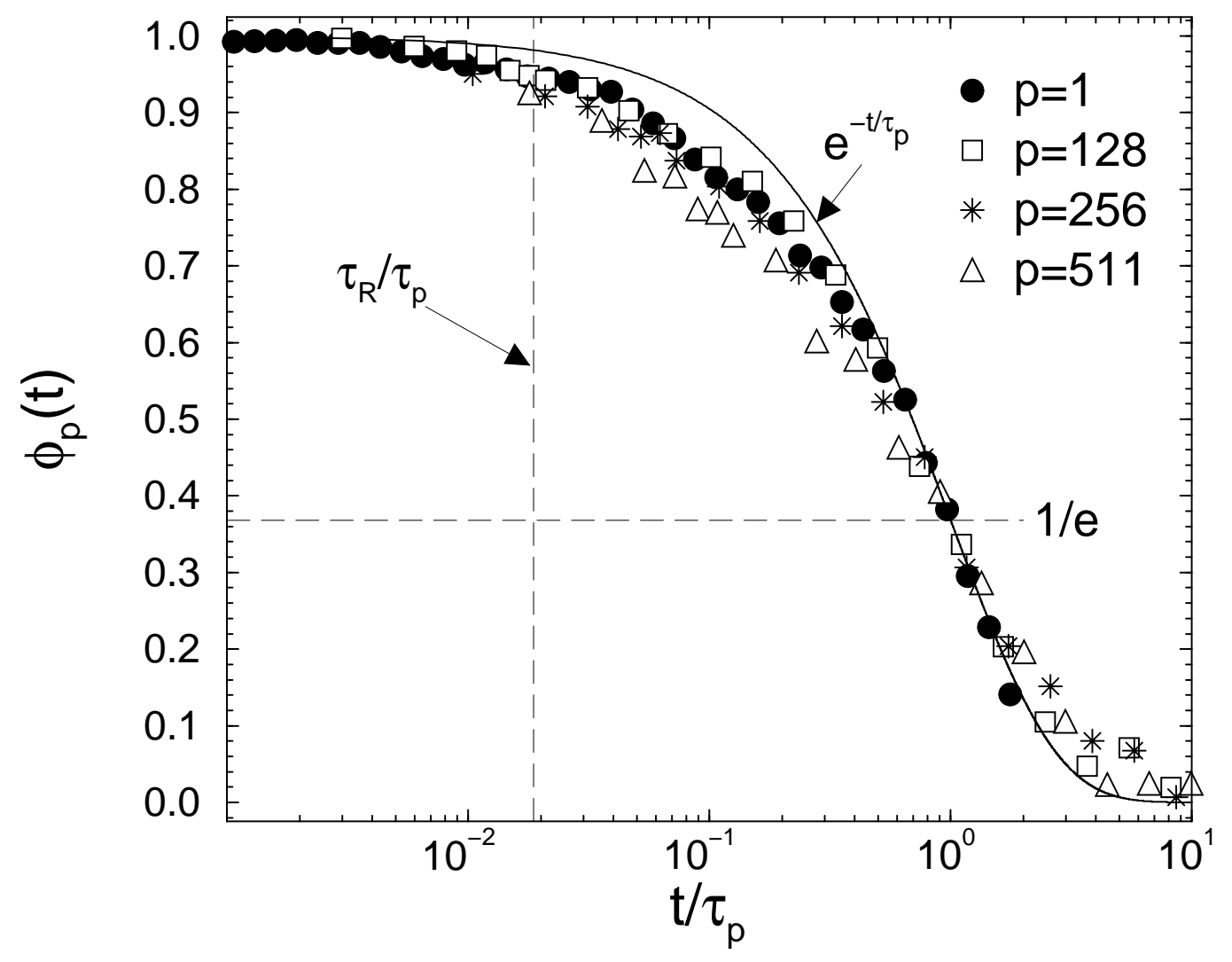

(b)

FIG. 12. 


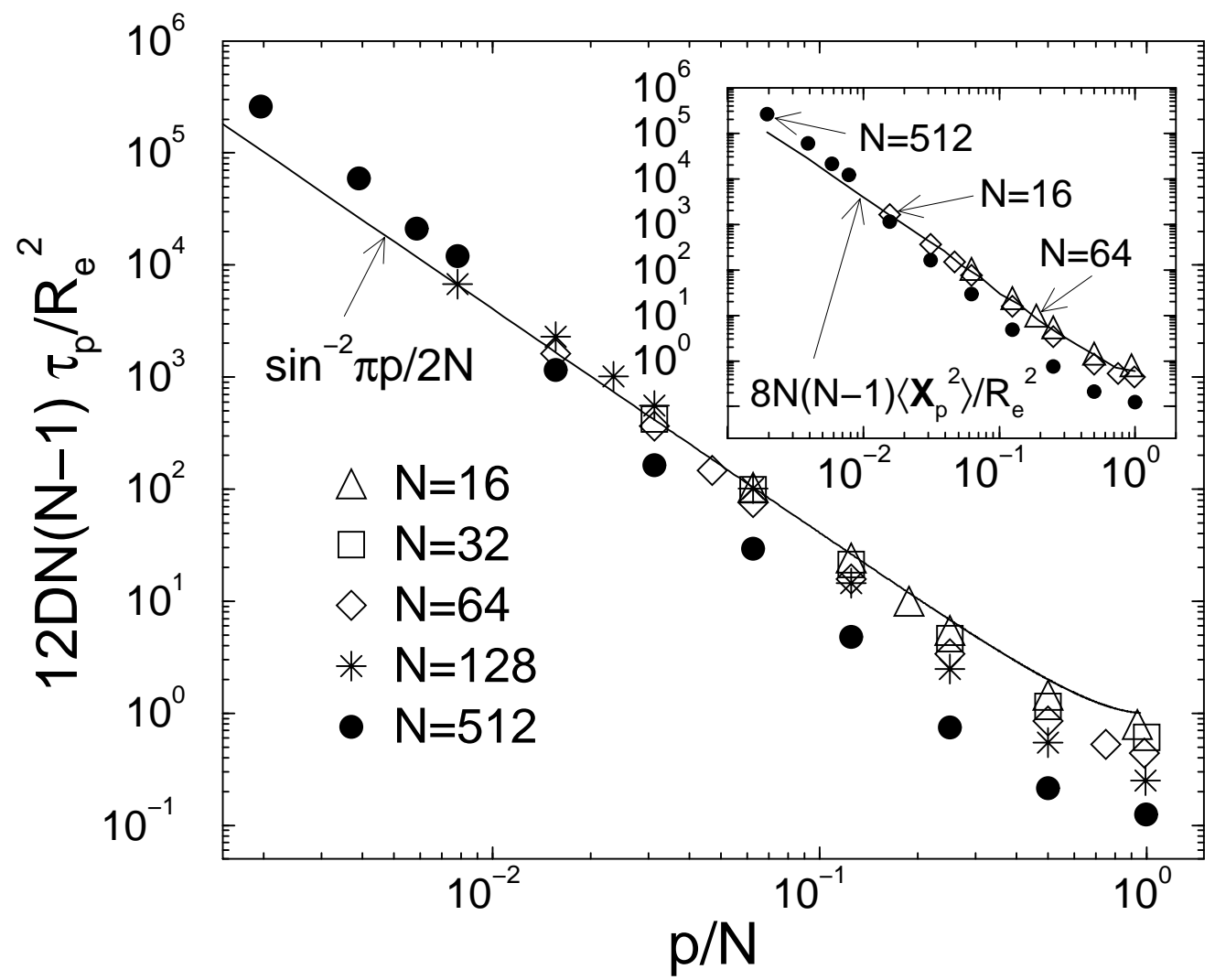

FIG. 13. 


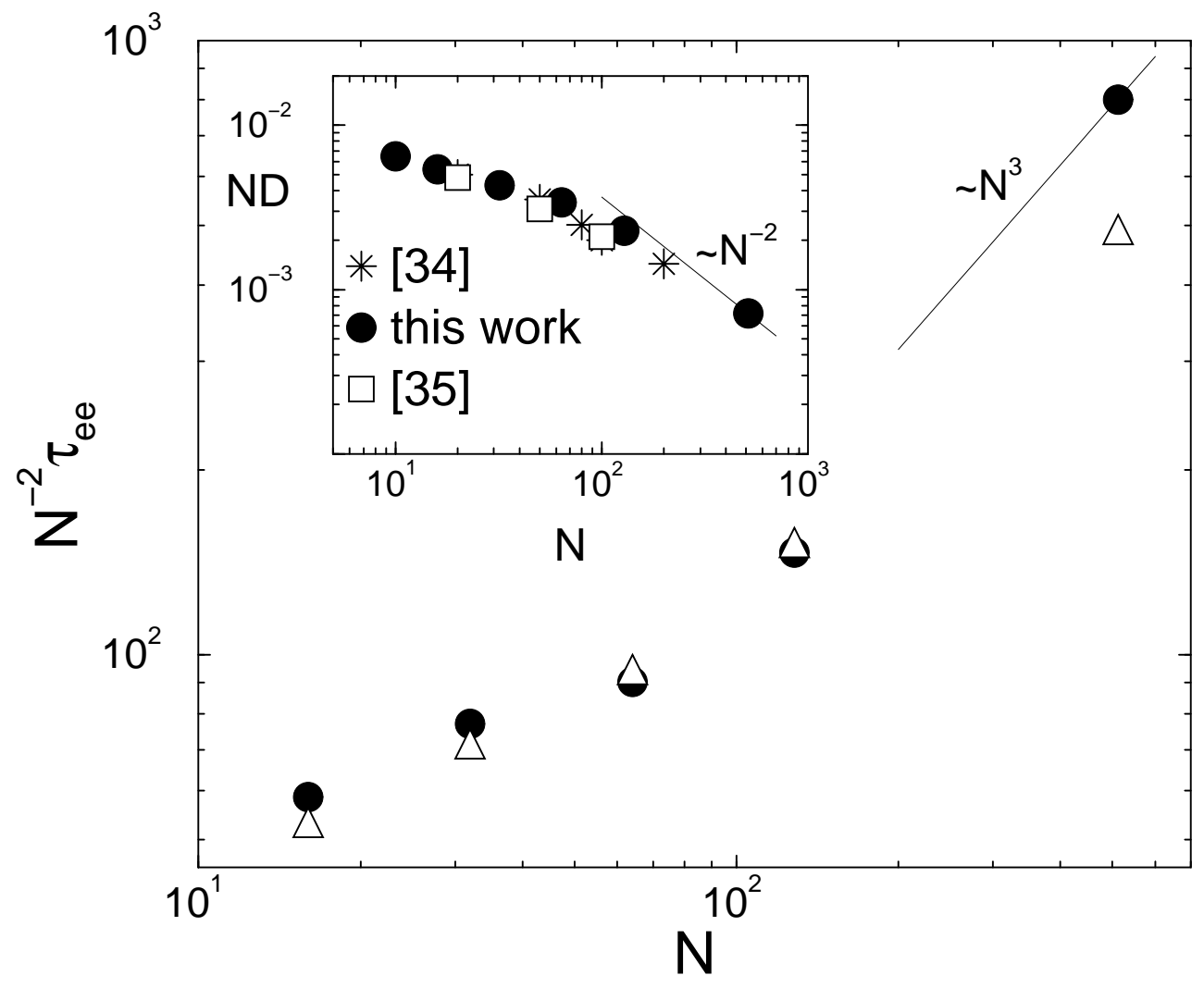

FIG. 14. 